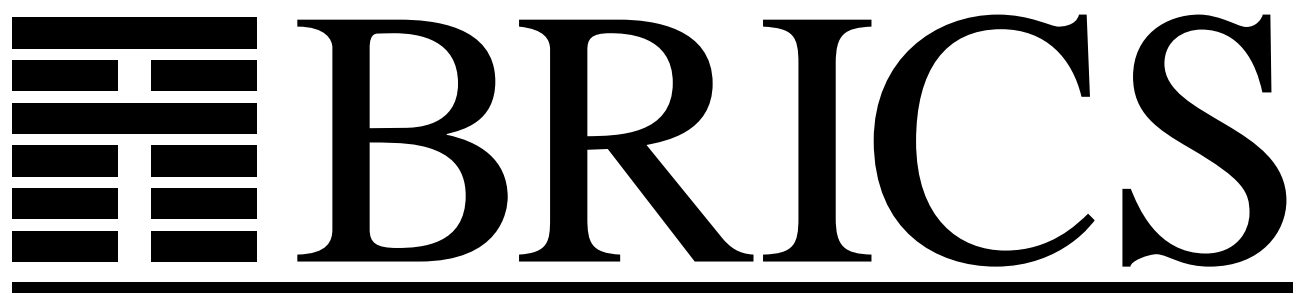

Basic Research in Computer Science

\title{
Bottom-Up $\beta$-Substitution:
}

Uplinks and $\lambda$-DAGs

Olin Shivers

Mitchell Wand 
Copyright (c) 2004, Olin Shivers \& Mitchell Wand.

BRICS, Department of Computer Science

University of Aarhus. All rights reserved.

Reproduction of all or part of this work is permitted for educational or research use on condition that this copyright notice is included in any copy.

See back inner page for a list of recent BRICS Report Series publications. Copies may be obtained by contacting:

\author{
BRICS \\ Department of Computer Science \\ University of Aarhus \\ Ny Munkegade, building 540 \\ DK-8000 Aarhus C \\ Denmark \\ Telephone: +4589423360 \\ Telefax: $\quad+4589423255$ \\ Internet: BRICS@brics.dk
}

BRICS publications are in general accessible through the World Wide Web and anonymous FTP through these URLs:

http://www.brics.dk

ftp: //ftp.brics.dk

This document in subdirectory RS / $04 / 38$ / 


\title{
Bottom-up $\beta$-reduction: uplinks and $\lambda$-DAGs (extended version)
}

\author{
Olin Shivers* \\ Georgia Institute of Technology \\ shivers@cc.gatech.edu
}

\author{
Mitchell Wand \\ Northeastern University \\ wand@ccs.neu.edu
}

December 31, 2004

\begin{abstract}
If we represent a $\lambda$-calculus term as a DAG rather than a tree, we can efficiently represent the sharing that arises from $\beta$-reduction, thus avoiding combinatorial explosion in space. By adding uplinks from a child to its parents, we can efficiently implement $\beta$-reduction in a bottom-up manner, thus avoiding combinatorial explosion in time required to search the term in a top-down fashion. We present an algorithm for performing $\beta$-reduction on $\lambda$-terms represented as uplinked DAGs; describe its proof of correctness; discuss its relation to alternate techniques such as Lamping graphs, explicit-substitution calculi and director strings; and present some timings of an implementation. Besides being both fast and parsimonious of space, the algorithm is particularly suited to applications such as compilers, theorem provers, and type-manipulation systems that may need to examine terms in-between reductions-i.e., the "readback" problem for our representation is trivial. Like Lamping graphs, and unlike director strings or the suspension $\lambda$ calculus, the algorithm functions by side-effecting the term containing the redex; the representation is not a "persistent" one. The algorithm additionally has the charm of being quite simple; a complete implementation of the data structure and algorithm is 180 lines of SML.
\end{abstract}

${ }^{*}$ Visiting faculty at BRICS, Department of Computer Science, University of Århus. 


\section{Contents}

1 Introduction 1

2 Guided tree substitution 2

3 Guiding tree search with uplinks 2

4 Upcopy with DAGs 3

5 Reduction on $\lambda$-DAGs $\quad 4$

$\begin{array}{lll}6 & \text { Fine points } & 7\end{array}$

7 Extended example $\quad 7$

8 Formal specification and correctness 9

9 Experiments $\quad 14$

10 Related work 16

10.1 Explicit-substitution calculi . . . . . . . . . . . . . . 16

10.2 Director strings . . . . . . . . . . . . . . . . . . 18

10.3 Optimal $\lambda$ reduction . . . . . . . . . . . . . . . . . 18

10.4 Two key issues: persistence and readback . . . . . . . . . . . . . . . 19

11 Other operations: cloning, equality and hashing 19

12 Possible variants and applications $\quad 20$

12.1 Cyclic graph structure . . . . . . . . . . . . . . . 20

12.2 Integrating with orthogonal implementation techniques . . . . . . . . 20

12.3 DAG-based compiler . . . . . . . . . . . . . . . . . . . . 21

12.4 Graph-based compiler . . . . . . . . . . . . . . . 21

13 Conclusion $\quad 21$

14 Acknowledgements $\quad 22$

$\begin{array}{ll}\text { References } & 22\end{array}$

$\begin{array}{lr}\text { A BetaSub.sml } & 25\end{array}$ 


\section{Introduction}

The $\lambda$ calculus $[2,5]$ is a simple language with far-reaching use in the programminglanguages and formal-methods communities, where it is frequently employed to represent, among other objects, functional programs, formal proofs, and types drawn from sophisticated type systems. Here, our particular interest is in the needs of client applications such as compilers, which may use $\lambda$-terms to represent both program terms as well as complex types. We are somewhat less focussed on the needs of graph-reduction engines, where there is greater representational license-a graph reducer can represent a particular $\lambda$-term as a chunk of machine code (e.g., by means of supercombinator extraction), because its sole focus is on executing the term. A compiler, in contrast, needs to examine, analyse and transform the term in-between operations on it, which requires the actual syntactic form of the term be available at the intermediate steps.

There are only three forms in the basic language: $\lambda$ expressions, variable references, and applications of a function to an argument:

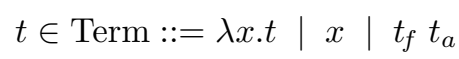

where $x$ stands for a member of some infinite set of variables. (We'll also allow ourselves parenthesisation of terms to indicate precedence in the parsing of concrete examples.)

Of the three basic operations on terms in the $\lambda$ calculus $-\alpha$-conversion, $\beta$-reduction, and $\eta$-reduction-it is $\beta$-reduction that accomplishes the "heavy lifting" of term manipulation. (The other two operations are simple to implement.) Unfortunately, naïve implementations of $\beta$-reduction can lead to exponential time and space blowup. $\beta$-reduction is the operation of taking an application term whose function subterm is a $\lambda$-expression, and substituting the argument term for occurrences of the $\lambda$ 's bound variable in the function body. The result, called the contractum, can be used in place of the original application, called the redex. We write

$$
(\lambda x . b) a \Rightarrow[x \mapsto a] b
$$

to express the idea that the redex applying function $\lambda x . b$ to argument $a$ reduces to the contractum $[x \mapsto a] b$, by which we mean term $b$, with free occurrences of $x$ replaced with term $a$.

We can define the core substitution function with a simple recursion:

$$
\begin{array}{llll}
{[y \mapsto t] \llbracket x \rrbracket} & =t & & x=y \\
{[y \mapsto t] \llbracket x \rrbracket} & =x & & x \neq y \\
{[x \mapsto t] \llbracket t_{f} t_{a} \rrbracket} & =\left([x \mapsto t] t_{f}\right)\left([x \mapsto t] t_{a}\right) & & \\
{[x \mapsto t] \llbracket \lambda y . b \rrbracket} & =\lambda y^{\prime} .\left([x \mapsto t]\left[y \mapsto y^{\prime}\right] b\right) & & y^{\prime} \text { fresh in } b \text { and } t .
\end{array}
$$

Note that, in the final case above, when we substitute a term $t$ under a $\lambda$-expression $\lambda y . b$, we must first replace the $\lambda$-expression's variable $y$ with a fresh, unused variable $y^{\prime}$ to ensure that any occurrence of $y$ in $t$ isn't "captured" by the $[x \mapsto t]$ substitution. If we know that there are no free occurrences of $y$ in $t$, this step is unnecessary-which is the case if we adopt the convention that every $\lambda$-expression binds a unique variable.

It is a straightforward matter to translate the recursive substitution function defined above into a recursive procedure. Consider the case of performing a substitution $[y \mapsto t]$ on an application $t_{f} t_{a}$. Our procedure will recurse on both subterms of the application... but we could also use a less positive term in place of "recurse" to indicate the trouble with the algorithmic handling of this case: search. In the case of an application, the procedure will blindly search both subterms, even though one or both may have no occurrences of the variable for which we search. Suppose, for example, that the function subterm $t_{f}$ is very large-perhaps millions of nodes-but contains no occurrences of the substituted variable $y$. The recursive substitution will needlessly search out the entire subterm, constructing an identical copy of $t_{f}$. What we want is some way to direct our recursion so that we don't waste time searching into subterms that do not contain occurrences of the variable being replaced. 


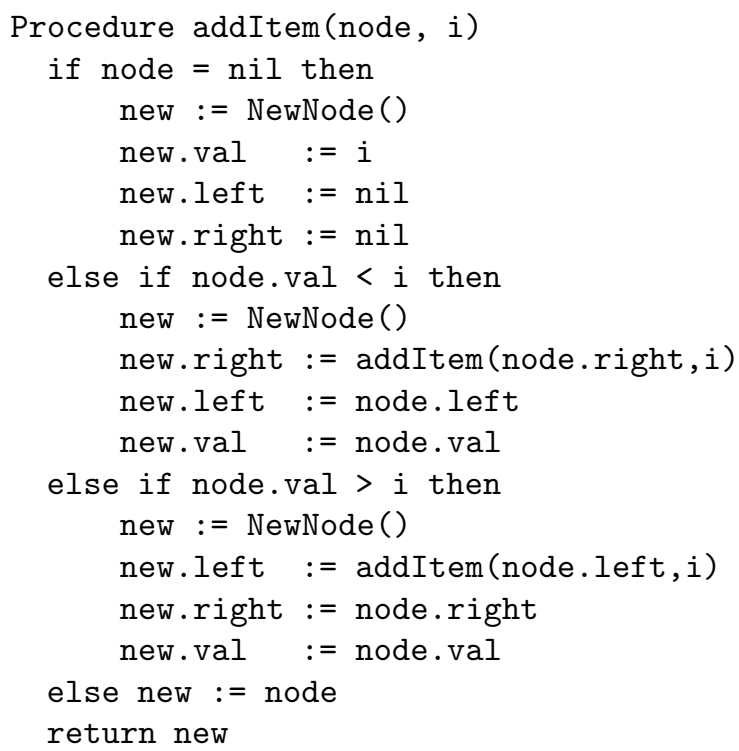

Figure 1: Make a copy of ordered binary tree node, with added entry $i$. The original tree is not altered.

\section{Guided tree substitution}

Let's turn to a simpler task to develop some intuition. Consider inserting an integer into a set kept as an ordered binary tree (Fig. 1). There are three things about this simple algorithm worth noting:

\section{- No search}

The pleasant property of ordered binary trees is that we have enough information as we recurse down into the tree to proceed only into subtrees that require copying.

\section{- Steer down; build up}

The algorithm's recursive control structure splits decision-making and the actual work of tree construction: the downward recursion makes the decisions about which nodes need to be copied, and the upward return path assembles the new tree.

\section{- Shared structure}

We copy only nodes along the spine leading from the targeted node to the root; the result tree shares as much structure as possible with the original tree.

\section{Guiding tree search with uplinks}

Unfortunately, in the case of $\beta$-reduction, there's no simple, compact way of determining, as we recurse downwards into a tree, which way to go at application nodes-an application has two children, and we might need to recurse into one, the other, both, or neither. Suppose, however, that we represent our tree using not only down-links that allow us to go from a parent to its children, but also with redundant up-links that allow us to go from a child to its parent. If we can (easily) find the leaf node in the original tree we wish to replace, we can chase uplinks along the spine from the old leaf to the tree root, copying as we go. This gives us the algorithm of Fig. 2, presented somewhat abstractly for simple binary trees. The core iteration of this algorithm is the $c \longmapsto c^{\prime}$ upcopy:<smiles>CO[Te]=CC12COP1O2</smiles> 


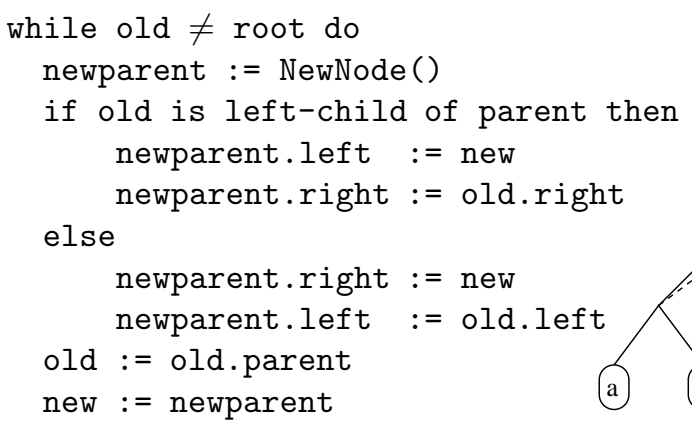

Figure 2: The algorithm copies a tree with child $\rightarrow$ parent uplinks, replacing leaf old with new. The example shows the algorithm making a copy of the original tree, replacing leaf $c$ with $x$. Arrows show the path of the algorithm as it copies up the spine of the tree from $c$ to the root; dotted lines show new structure.

We take a child $c$ and its intended replacement $c^{\prime}$, and replicate the parent $p$ of $c$, making the $c \mapsto c^{\prime}$ substitution. This produces freshly-created node $p^{\prime}$; we can now iterate, doing a $p \mapsto p^{\prime}$ upcopy into the parent of $p$ at the next step, and so on, moving up through the original tree until we reach the root.

Note the similar properties this upcopy algorithm has with the previous algorithm: no search required; we build as we move upwards; we share as much structure as possible with the old tree, copying only the nodes along the "spine" leading from the leaf up to the root. For a balanced tree, the amount of copying is logarithmic in the total number of nodes. By starting at the leaf node to be replaced in the old tree, the construction phase just follows uplinks to the root, instead of using a path saved in the recursion stack by the downwards search.

\section{Upcopy with DAGs}

We can avoid space blowup when performing $\beta$-reduction on $\lambda$-calculus terms if we can represent them as directed acyclic graphs (DAGs), not trees. Allowing sharing means that when we substitute a large term for a variable that has five or six references inside its binding $\lambda$-expression, we don't have to create five or six distinct copies of the term (that is, one for each place it occurs in the result). We can just have five or six references to the same term. This has the potential to provide logarithmic compression on the simple representation of $\lambda$-calculus terms as trees. These term DAGs can be thought of as essentially a space-saving way to represent term trees, so we can require them, like trees, to have a single top or root node, from which all other nodes can be reached.

When we shift from trees to DAGs, however, our simple functional upcopy algorithm no longer suffices: we have to deal with the fact that there may be multiple paths from a leaf node (a variable reference) of our DAG up to the root of the DAG. That is, any term can have multiple parents. However, we can modify our upwards-copying algorithm in the standard way one operates on DAGs: we search upwards along all possible paths, marking nodes as we encounter them. The first time we copy up into a node $n$, we replicate it, as in the previous tree algorithm, and continue propagating the copy operation up the tree the (possibly multiple) parents of $n$. However, before we move upwards from $n$, we first store the copy $n^{\prime}$ away in a "cache" field of $n$. If we later copy up into $n$ via its other child, the presence of the copy $n^{\prime}$ in the cache slot of $n$ will signal the algorithm that it should not make a second copy of $n$, and should not proceed upwards from $n$ - that has already been handled. Instead, it mutates the existing copy $n^{\prime}$ and returns immediately.

The code to copy a binary DAG, replacing a single leaf, is shown in Fig. 3. Every node in the DAG maintains a set of its uplinks; each uplink is represented as a 〈parent, relation 


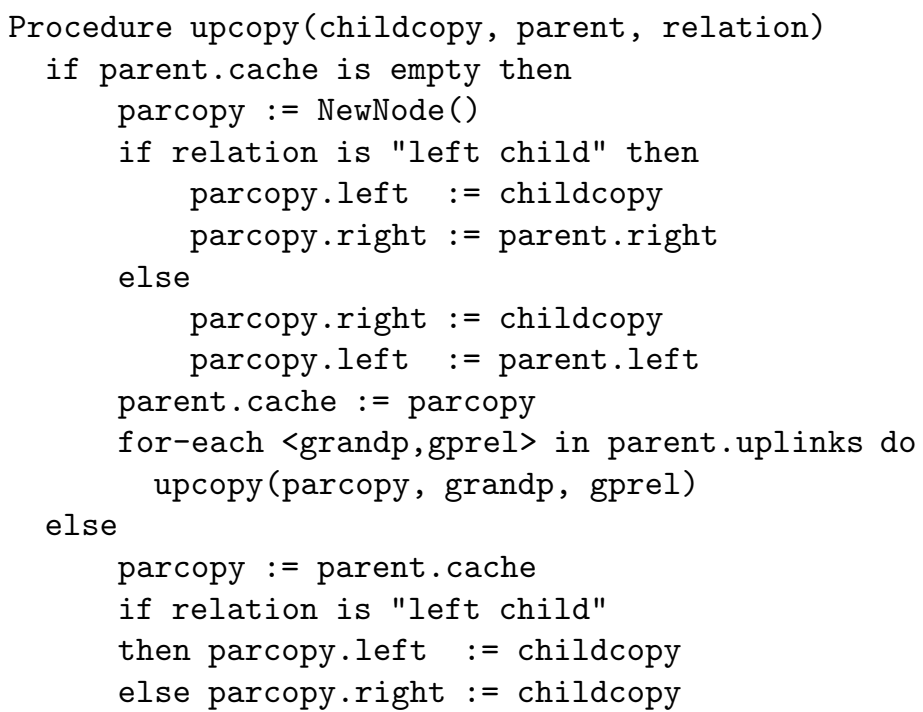

Figure 3: Procedure upcopy makes a copy of a binary DAG, replacing the relation child (left or right) of parent with childcopy.

pair. For example, if node $c$ is the left child of node $p$, then the pair $\langle p$, left-child $\rangle$ will be one of the elements in $c$ 's uplink set.

The upcopy algorithm explores each edge on all the paths between the root of the DAG and the replaced leaf exactly once; marking parent nodes by depositing copies in their cache slots prevents the algorithm from redundant exploration. Hence this graph-marking algorithm runs in time proportional to the number of edges, not the number of paths (which can be exponential in the number of edges). Were we to "unfold" the DAG into its equivalent tree, we would realise this exponential blowup in the size of the tree, and, consequently, also in the time to operate upon it. Note that, analogously to the tree-copying algorithm, the new DAG shares as much structure as possible with the old DAG, only copying nodes along the spine (in the DAG case, spines) from the replaced leaf to the root.

After an upcopy has been performed, we can fetch the result DAG from the cache slot of the original DAG's root. We must then do another upwards search along the same paths to clear out the cache fields of the original nodes that were copied, thus resetting the DAG for future upcopy operations. (Alternatively, we can keep counter fields on the nodes to discriminate distinct upcopy operations, and perform a global reset on the term when the current-counter value overflows.) This cache-clearing pass, again, takes time linear in the number of edges occurring on the paths from the copied leaf to the root:

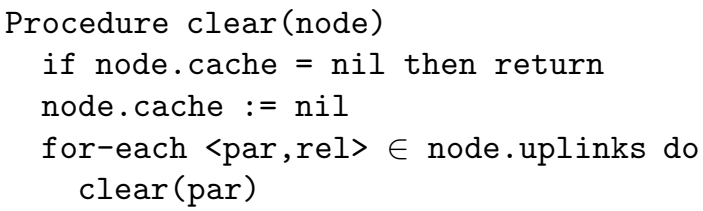

\section{Reduction on $\lambda$-DAGs}

We now have the core idea of our DAG-based $\beta$-reduction algorithm in place, and can fill in the details specific to our $\lambda$-expression domain.

Basic representation We will represent a $\lambda$-calculus term as a rooted DAG. 
Sharing Sharing will be generally allowed, and sharing will be required of variablereference terms. That is, any given variable will have no more than one node in the DAG representing it. If one variable is referenced by (is the child of) multiple parent nodes in the graph, these nodes simply will all contain pointers to the same data structure.

Bound-variable short-cuts Every $\lambda$-expression node will, in addition to having a reference to its body node, also have a reference to the variable node that it binds. This, of course, is how we navigate directly to the leaf node to replace when we begin the upcopy for a $\beta$-reduction operation. Note that this amounts to an $\alpha$-uniqueness condition-we require that every $\lambda$-expression bind a unique variable.

Cache fields Every application node has a cache field that may either be empty or contain another application node. $\lambda$-expression nodes do not need cache fields-they only have one child (the body of the $\lambda$-expression), so the upcopy algorithm can only copy up through a $\lambda$-expression once during a $\beta$-reduction.

Uplinks Uplinks are represented by 〈parent, relation $\rangle$ pairs, where the three possible relations are " $\lambda$ body," "application function," and "application argument." For example, if a node $n$ has an uplink $\langle l, \lambda$-body $\rangle$, then $l$ is a $\lambda$-expression, and $n$ is its body.

Copying $\lambda$-expressions With all the above structure in place, the algorithm takes shape. To perform a $\beta$-reduction of redex $(\lambda x . b) a$, where $b$ and $a$ are arbitrary subterms, we simply initiate an $x \mapsto a$ upcopy. This will copy up through all the paths connecting top node $b$ and leaf node $x$, building a copy of the DAG with $a$ in place of $x$, just as we desire.

Application nodes, having two children, are handled just as binary-tree nodes in the general DAG-copy algorithm discussed earlier: copy, cache \& continue on the first visit; mutate the cached copy on a second visit. $\lambda$-expression nodes, however, require different treatment. Suppose, while we are in the midst of performing the reduction above, we find ourselves performing a $c \mapsto c^{\prime}$ upcopy, for some internal node $c$, into a $\lambda$ parent of $c: \lambda y . c$. The general structure of the algorithm calls for us to make a copy of the $\lambda$-expression, with body $c^{\prime}$. But we must also allocate a fresh variable $y^{\prime}$ for our new $\lambda$-expression, since we require all $\lambda$-expressions to bind distinct variables. This gives us $\lambda y^{\prime} . c^{\prime}$. Unfortunately, if old body $c$ contains references to $y$, these will also occur in $c^{\prime}$-not $y^{\prime}$. We can be sure $c^{\prime}$ contains no references to $y^{\prime}$, since $y^{\prime}$ was created after $c^{\prime}$ ! We need to fix up body $c^{\prime}$ by replacing all its references to $y$ with references to $y^{\prime}$.

Luckily, we already have the mechanism to do this: before progressing upwards to the parents of $\lambda y . c$, we simply initiate a $y \mapsto y^{\prime}$ upcopy through the existing DAG. This upcopy will proceed along the paths leading from the $y$ reference, up through the DAG, to the $\lambda y . c$ node. If there are such paths, they must terminate on a previously-copied application node, at which point the upcopy algorithm will mutate the cached copy and return.

Why must these paths all terminate on some previously copied application node? Because we have already traversed a path from $x$ up to $\lambda y . c$, copying and caching as we went. Any path upwards from the $y$ reference must eventually encounter $\lambda y . c$, as well-this is guaranteed by lexical scope. The two paths must, then, converge on a common application node- the only nodes that have two children. That node was copied and cached by the original $x$-to- $\lambda y$. $c$ traversal.

When the $y \mapsto y^{\prime}$ upcopy finishes updating the new DAG structure and returns, the algorithm resumes processing the original $c \mapsto c^{\prime}$ upcopy, whose next step is to proceed upwards with a $(\lambda y . c) \mapsto\left(\lambda y^{\prime} . c^{\prime}\right)$ upcopy to all of the parents of $\lambda y . c$, secure that the $c^{\prime}$ sub-DAG is now correct.

The single-DAG requirement We've glossed over a limitation of the uplink representation, which is that a certain kind of sharing is not allowed: after a $\beta$-reduction, the original redex must die. That is, the model we have is that we start with a $\lambda$-calculus term, represented as a DAG. We choose a redex node somewhere within this DAG, reduce it, and 
alter the original DAG to replace the redex with the contractum. When done, the original term has been changed: where the redex used to be, we now find the contractum. What we can't do is choose a redex, reduce it, and then continue to refer to the redex or maintain an original, unreduced copy of the DAG. Contracting a redex kills the redex; the term data structure is not "pure functional" or "persistent" in the sense of the old values being unchanged. (As we discuss later, we can, however, "clone" a multiply-referenced redex, splitting the parents between the original and the clone, and then contract only one of the redexes.)

This limitation is due to the presence of the uplinks. They mean that a subterm can belong to only one rooted DAG, in much the same way that the backpointers in a doublylinked list mean that a list element can belong to only one list (unlike a singly-linked list, where multiple lists can share a common tail). The upcopy algorithm assumes that the uplinks exactly mirror the parent $\rightarrow$ child downlinks, and traces up through all of them. This rules out the possibility of having a node belong to multiple distinct rooted DAGs, such as a "before" and "after" pair related by the $\beta$-reduction of some redex occurring within the "before" term.

Hence the algorithm, once it has finished the copying phase, takes the final step of disconnecting the redex from its parents, and replacing it with the contractum. The redex application node is now considered dead, since it has no parents, and can be removed from the parent/uplink sets of its children and deallocated. Should one of its two children thus have its parent set become empty, it, too, can be removed from the parent sets of its children and deallocated, and so forth. Thus we follow our upwards-recursive construction phase with a downwards-recursive deallocation phase.

It's important to note that this deallocation phase is not optional. A dead node must be removed from the parent sets of its children, lest we subsequently waste time doing an upcopy from a child up into a dead parent during a later reduction. Failing to deallocate dead nodes would also break the invariants of the data structure, such as the requirement that uplinks mirror the downlink structure, or the fact that every path upwards from a variable reference must encounter that variable's binding $\lambda$-node.

Termination and the top application Another detail we've not yet treated is termination of the upcopy phase. One way to handle this is simply to check as we move up through the DAG to see if we've arrived at the $\lambda$-expression being reduced, at which point we could save away the new term in some location and return without further upward copying. But there is an alternate way to handle this. Suppose we are contracting $\operatorname{redex}(\lambda x . b) n$, for arbitrary sub-terms $b$ and $n$. At the beginning of the reduction operation, we first check to see if $x$ has no references (an easy check: is its uplink set empty?). If so, the answer is $b$; we are done.

Otherwise, we begin at the $\lambda$-expression being reduced and scan downwards from $\lambda$ expression to body, until we encounter a non- $\lambda$-expression node-that is, a variable or an application. If we halt at a variable, it must be $x$-otherwise $x$ would have no references, and we've already ruled that out. This case can also be handled easily: we simply scan back through this chain of nested $\lambda$-expressions, wrapping fresh $\lambda$-expressions around $n$ as we go.

Finally, we arrive at the general case: the downward scan halts at the topmost application node $a$ of sub-term $b$. We make an identical copy $a^{\prime}$ of $a$, i.e. one that shares both the function and argument children, and install $a^{\prime}$ in the cache slot of $a$.

Now we can initiate an $x \mapsto n$ upcopy, knowing that all upwards copying must terminate on a previously-copied application node. This is guaranteed by the critical, key invariant of the DAG: all paths from a variable reference upward to the root must encounter the $\lambda$-node binding that variable-this is simply lexical scoping in the DAG context. The presence of $a^{\prime}$ in the cache slot of $a$ will prevent upward copying from proceeding above $a$. Node $a$ acts as a sentinel for the algorithm; we can eliminate the root check from the upcopy code, for time savings. 
When the upcopy phase finishes, we pass $a^{\prime}$ back up through the nested chain of $\lambda$ expressions leading from $a$ back to the top $\lambda x . b$ term. As we pass back up through each $\lambda$-expression $\lambda y$.t, we allocate a fresh $\lambda$-expression term and a fresh variable $y^{\prime}$ to wrap around the value $t^{\prime}$ passed up, then perform a $y \mapsto y^{\prime}$ upcopy to fix up any variable references in the new body, and then pass the freshly-created $\lambda y^{\prime} . t^{\prime}$ term on up the chain.

(Note that the extended example shown in Sec. 7 omits this technique to simplify the presentation.)

\section{Fine points}

These fine points of the algorithm can be skipped on a first reading.

Representing uplinks A node keeps its uplinks chained together in a doubly-linked list, which allows us to remove an uplink from a node's uplink set in constant time. We will need to do this, for example, when we mutate a previously copied node $n$ to change one of its children - the old child's uplink to $n$ must be removed from its uplink set.

We simplify the allocation of uplinks by observing that each parent node has a fixed number of uplinks pointing to it: two in the case of an application and one in the case of a $\lambda$-expression. Therefore, we allocate the uplink nodes along with the parent, and thread the doubly-linked uplink lists through these pre-allocated nodes.

An uplink doubly-linked list element appears in the uplink list of the child, but the element belongs to the parent. For example, when we allocate a new application node, we simultaneously allocate two uplink items: one for the function-child uplink to the application, and one for the argument-child uplink to the application. These three data structures have identical lifetimes; the uplinks live as long as the parent node they reference. We stash them in fields of the application node for convenient retrieval as needed. When we mutate the application node to change one of its children, we also shift the corresponding uplink structure from the old child's uplink list to the new child's uplink list, thus keeping the uplink pointer information consistent with the downlink pointer information.

The single-reference fast path Consider a redex $(\lambda x . b) n$, where the $\lambda$-expression being reduced has exactly one parent. We know what that parent must be: the redex application itself. This application node is about to die, when all references to it in the term DAG are replaced by references to the contractum. So the $\lambda$-expression itself is about to become completely parentless-i.e., it, too, is about to die. This means that any node on a path from $x$ up to the $\lambda$-expression will also die. Again, this is the key invariant provided by lexical scope: all paths from a variable reference upward to the root must encounter the $\lambda$-expression binding that variable. So if the $\lambda$-expression has no parents, then all paths upwards from its variable must terminate at the $\lambda$-expression itself.

This opens up the possibility of an alternate, fast way to produce the contractum: when the $\lambda$-expression being reduced has only one parent, mutate the $\lambda$-expression's body, altering all of $x$ 's parents to refer instead to $n$. We do no copying at all, and may immediately take the $\lambda$-expression's body as our answer, discarding the $\lambda$-expression and its variable $x$ (in general, a $\lambda$-expression and its variable are always allocated and deallocated together).

Opportunistic iteration The algorithm can be implemented so that when a node is sequencing through its list of uplinks, performing a recursive upcopy on each one, the final upcopy can be done with a tail recursion (or, if coded in a language like $\mathrm{C}$, as a straight iteration). This means that when there is no sharing of nodes by parents, the algorithm tends to iteratively zip up chains of single-parent links without pushing stack frames.

\section{Extended example}

We can see the sequences of steps taken by the algorithm on a complete example in Fig. 4. Part 4(a) shows the initial redex, which is $(\lambda x .(x(\lambda y . x(u y)))(\lambda y . x(u y))) t$, where the 


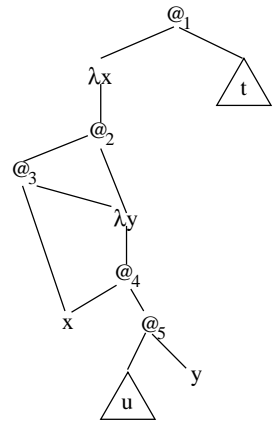

(a)

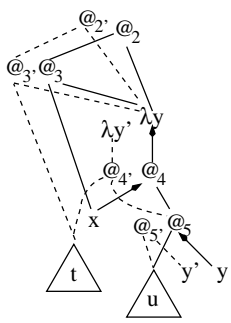

(f)

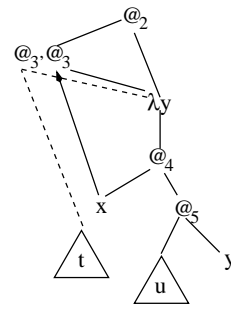

(b)

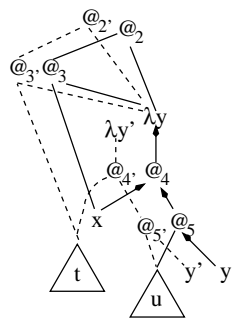

(g)

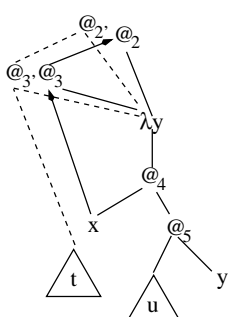

(c)

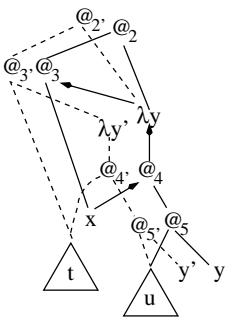

(h)

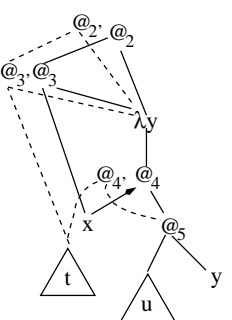

(d)

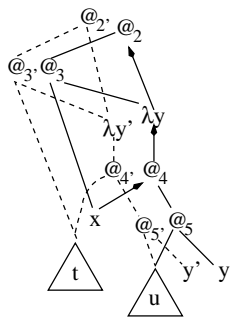

(i)

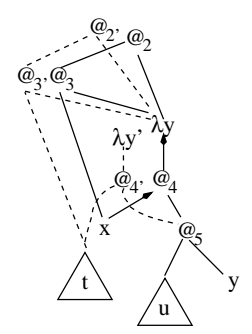

(e)

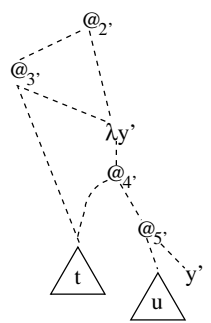

(j)

Figure 4: A trace of a bottom-up reduction of term $(\lambda x .(x(\lambda y \cdot x(u y)))(\lambda y \cdot x(u y))) t$, where the $(\lambda y . x(u y))$ term is shared, and sub-terms $t$ and $u$ are not specified.

$(\lambda y . x(u y))$ subterm is shared, and $t$ and $u$ are arbitrary, unspecified subterms with no free occurrences of $x$ or $y$. To help motivate the point of the algorithm, imagine that the subterms $t$ and $u$ are enormous - things we'd like to avoid copying or searching - and that the $\lambda x$ node has other parents besides application 1 , so we cannot blindly mutate it without corrupting what the other parents see. (If the $\lambda x$ node doesn't have other parents, then the single-reference fast-path described in the previous section applies, and we are allowed to mutate the term, for a very fast reduction.)

In the following subfigure, 4(b), we focus in on the body of the $\lambda$-expression being reduced. We iterate over the parents of its variable reference $x$, doing an $x \mapsto t$ upcopy; this is the redex-mandated substitution that kicks off the entire reduction. The first parent of $x$ is application 3, which is copied, producing application $3^{\prime}$, which has function child $t$ instead of the variable reference $x$, but has the same argument child as the original application 3 , namely the $\lambda y$ term. Dotted lines show new DAG structure; arrowheads on old structure indicate the recursive control flow of the algorithm's upcopy steps. The copy $3^{\prime}$ is saved away in the cache slot of application 3 , in case we upcopy into 3 from its argument child in the future.

Once we've made a copy of a parent node, we must recursively perform an upcopy for it. That is, we propagate a $3 \mapsto 3^{\prime}$ upcopy to the parents of application 3 . There is only one such parent, application 2 . In subfigure $4(\mathrm{c})$, we see the result of this upcopy: the application $2^{\prime}$ is created, with function child $3^{\prime}$ instead of 3 ; the argument child, $\lambda y$, is carried over from the original application 2. Again, application $2^{\prime}$ is saved away in the cache slot of application 2 .

Application 2 is the root of the upcopy DAG, so once it has been copied, control returns to application 3 and its $3 \mapsto 3^{\prime}$ upcopy. Application 3 has only one parent, so it is done. 
Control returns to $x$ and its $x \mapsto t$ upcopy, which proceeds to propagate upwards to the second parent of $x$, application 4 .

We see the result of copying application 4 in subfigure $4(d)$. The new node is $4^{\prime}$, which has function child $t$ where 4 has $x ; 4^{\prime}$ shares its argument child, application 5, with application 4 . Once again, the copy $4^{\prime}$ is saved away in the cache slot of application 4 .

Having copied application 4 , we recursively trigger a $4 \mapsto 4^{\prime}$ upcopy, which proceeds upwards to the sole parent of application 4. We make a copy of $\lambda y$, allocating a fresh variable $y^{\prime}$, with the new body $4^{\prime}$. This is shown in subfigure 4(e).

Since the new $\lambda y^{\prime}$ term binds a fresh variable, while processing the $\lambda y$ term we must recursively trigger a $y \mapsto y^{\prime}$ upcopy, which begins in subfigure 4(f). We iterate through the parents of variable reference $y$, of which there is only one: application 5 . This is copied, mapping child $y$ to replacement $y^{\prime}$ and sharing function child $u$. The result, $5^{\prime}$, is saved away in the cache slot of application 5 .

We then recursively trigger a $5 \longmapsto 5^{\prime}$ upcopy through the parents of application 5 ; there is only one, application 4. Upon examining this parent (subfigure 4(g)), we discover that 4 already has a copy, $4^{\prime}$, occupying its cache slot. Rather than create a second, new copy of 4 , we simply mutate the existing copy so that its argument child is the new term $5^{\prime}$. Mutating rather than freshly allocating means the upcopy proceeds no further; responsibility for proceeding upwards from 4 was handled by the thread of computation that first encountered it and created $4^{\prime}$. So control returns to application 5 , which has no more parents, and then to $y$, who also has no more parents, so control finally returns to the $\lambda y$ term that kicked off the $y \mapsto y^{\prime}$ copy back in subfigure 4(f).

In subfigure 4(h), the $\lambda y$ term, having produced its copy $\lambda y^{\prime}$, continues the upcopy by iterating across its parents, recursively doing a $\lambda y \mapsto \lambda y^{\prime}$ upcopy. The first such parent is application 3 , which has already been copied, so it simply mutates its copy to have argument child $\lambda y^{\prime}$ and returns immediately.

The second parent is application 2 , which is handled in exactly the same way in subfigure 4(i). The $\lambda y$ term has no more parents, so it returns control to application 4 , who has no more parents, and so returns control to variable reference $x$. Since $x$ has no more parents, we are done. The answer is application $2^{\prime}$, which is shown in subfigure 4(j). We can change all references to application 1 in the DAG to point, instead, to application $2^{\prime}$, and then deallocate 1 . Depending on whether or not the children of application 1 have other parents in the DAG, they may also be eligible for deallocation. This is easily performed with a downwards deallocation pass, removing dead nodes from the parent lists of their children, and then recursing if any child thus becomes completely parentless.

\section{Formal specification and correctness}

In this section, we will more formally specify the core of the bottom-up reduction algorithm. A precise, fully-detailed argument establishing its correctness is beyond the scope of this paper; we provide only the structural skeleton, eliding details and simplifying the formalisations, and rely on the reader to interpolate where necessary.

We proceed by defining the essential core of the algorithm as a pair of math functions, about which we can reason; this definition strips away details of the data-structures (such as the use of doubly-linked lists, etc.) in favor of using simple mathematical structures such as sets, graphs and partial functions. We will take $\lambda$-terms to be graphs: a term is a finite DAG with labelled edges and nodes. Every vertex is labelled as either a $\lambda$, an application, or a variable-reference vertex. We will frequently use $l$ for $\lambda$ vertices, $a$ for applications, $x, y$ for variable vertices, and $n$ for general vertices. Edges are labelled with one of $\{$ body, fun, arg, bvar\}. We will frequently use a "dotted accessor" notation, e.g., writing $l$.body for the vertex at the end of the (presumed unique) body edge beginning at node $l$. A $\lambda$-node is connected to its body by a body edge; and to its bound variable by a bvar edge. An application node is connected to its function by a fun edge; and to its argument by an arg edge. We assume enough constraints on labelling and graph structure to 
preserve the syntactic structure of the $\lambda$ calculus, $e . g$., the out-degree of a variable-reference node is zero. We also assume a lexical-scoping resolution of variable reference, i.e., that binding dominates reference: any path from the root to a variable vertex must go through the $\lambda$ vertex binding that variable.

Define $\Lambda_{\text {dag }}$ to be the set of DAGs satisfying these constraints. The classic $\lambda$ calculus is defined in terms of trees; define $\Lambda_{\text {tree }}$ to be the standard realisation of $\lambda$-terms as abstractgrammar trees, with variables drawn from the variable-labelled vertices of $\Lambda_{\text {dag }}$. The idea behind our DAGs is that they are simply a compact representation for these trees, so we should define the "meaning" of a DAG simply to be the tree to which it "unfolds." We specify this by defining a DAG-to-tree conversion function, unfold:

$$
\begin{aligned}
\operatorname{unfold}(g, x)= & x \\
\operatorname{unfold}(g, a)= & \operatorname{unfold}(g, a . \text { fun }) @ \operatorname{unfold}(g, a \cdot \arg ) \\
\operatorname{unfold}(g, l)= & \lambda x . \operatorname{unfold}(g, l . \text { body }) \\
& \text { where } x=\text { l.bvar }
\end{aligned}
$$

(Here, we take " $n_{1} @ n_{2}$ " to mean the tree whose root is an application node with function child $n_{1}$ and argument child $n_{2}$.) This recursively-defined function is well-defined, as unfold's domain of finite DAGS is well ordered.

The unfold function helps makes precise the correctness requirement for our algorithm: reducing a subterm in a DAG, and then unfolding the result, should be the same as unfolding the original DAG and then reducing the corresponding subterms in the tree. Notice that if a redex in a DAG has multiple parents (or, generally, multiple paths to the root), then there will be multiple corresponding redexes in the unfolded tree; reducing this redex in the DAG is equivalent to reducing all of the corresponding redexes in the tree.

In order to connect the state of the graph at intermediate points in the algorithm to the hoped-for final result, we also need to define a "predictive unfold" function:

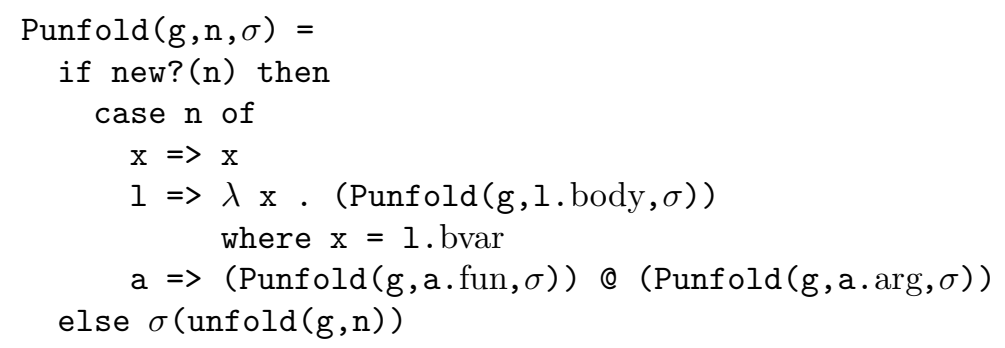

Punfold takes a graph, a root vertex $n$, and a substitution $\sigma$. We model a substitution as a partial function from (variable) nodes to $\Lambda_{\text {tree }}$ terms:

$$
\sigma \in \text { Subst }=\text { Node } \rightarrow \Lambda_{\text {tree }}
$$

Punfold has two "modes," depending on whether it is given old graph structure (that is, pre-reduction), or new graph structure (that is, created as part of the possibly on-going reduction process). Punfold recursively unfolds new structure, but when it traces into old structure, it unfolds and then applies $\sigma$ to the old sub-DAG-that is, Punfold "predicts" what the algorithm will do when it gets to that sub-DAG. We can answer Punfold's old/new question by assuming the original, pre-reduction graph is available; we'll refer to this graph as $g_{0}$. (Note that Punfold passes $\sigma$ down past binding occurrences as it recurses. This is safe to do, as we will restrict the domain of $\sigma$ to be old structure, so that passing these substitutions into the scope of forms that bind new variables cannot cause capture.)

With these descriptive tools in place, we can now analyse the algorithm's execution. $\operatorname{Punfold}\left(g_{0}, b,[x \mapsto n]\right)$ produces the contractum for DAG-redex $(\lambda x . b) n$. Further, the key algorithmic invariant involves Punfold, which will produce the same result throughout the processing of the reduction - the predictive part of Punfold covers parts of the DAG the algorithm has not yet processed. But as the algorithm progresses, Punfold will do less and 
less prediction, and more and more simple unfolding. By the end of the algorithm, Punfold will do no prediction at all (that is, no element of old structure will lay within the domain of the initial substitution), so that Punfold will be equivalent to unfold on the final result. So, we may conclude that unfolding the final result is what we wanted.

This, coupled with a progress result to guarantee termination on a finite DAG, provides us a correctness claim for the algorithm.

Fig. 5 presents the core algorithm as a pair of mathematical functions: Sub, which implements substitution, and uc, which implements the upcopy operation; they are defined in the same sort of "pseudo functional programming language" style we used for Punfold. (Note that the auxiliary function, iter, to be well-defined, must remove elements from $S$ in some determined order.) We write parlinks $\left(\right.$ old,$\left.g_{0}\right)$ to mean the set of parent/edge-label pairs describing old's uplinks in the original graph $g_{0}$. We use the expression "freshnode label" to mean the selection of a fresh vertex, labelled label, that is not occurring in the implied current graph.

The algorithm operates on graphs $g$ that are represented by a quadruple $(N, E, \rho, T)$ of labelled nodes, labelled edges, cache $\rho$, and visited-edge set $T$; the cache stores the mapping from old, copied nodes to their new nodes, as they are created by the upcopy steps. The cache represents the collective behaviour of the cache slot in the algorithm. We model these with finite, partial functions from nodes to nodes:

$$
\rho \in \text { Cache }=\text { Node } \rightarrow \text { Node } .
$$

Where the algorithm stores an item $n^{\prime}$ in the cache field of some node $n$, the mathematical function adds an $\left(n, n^{\prime}\right)$ entry to the current cache $\rho$. (Note that the cache always maps old nodes, from $g_{0}$, to new nodes.) To aid the statement of invariants, the abstract algorithm caches the old $\mapsto$ new translations for all copied nodes, not just application nodes (as the actual algorithm does). We use dotted-accessor notation, $g . \rho$, to write the cache of a given graph $g$.

The functions modelling the algorithm track extra values ( $\sigma$, ip, and $g . T)$ that are not present in the algorithm. These extra values are needed to state the key pre- and postconditions of the functions; they do not contribute to the functions' final value, and so can be omitted from an actual implementation. The important pre- and post-conditions for Sub and uc are:

- $\mathrm{CG}[\sigma, g]$

"Cache is good." Entries in the cache are related by Punfold: if we see an $\left(n, n^{\prime}\right)$ entry in the cache $g . \rho$, then $n^{\prime}$ represents a substitution that has been, or is in the midst of being, performed on $n$. That is, $\operatorname{Punfold}\left(g, n^{\prime}, \sigma\right) \stackrel{\alpha}{=} \sigma$ unfold $(g, n)$. Because the substitution may not be complete, we must unfold $n^{\prime}$ with the predictive unfolder, which will interpolate the uncompleted parts of the substitution into the result tree for us. The reason this relation needs a general substitution is because of the extra copying cascades triggered when the algorithm copies up through a $\lambda$-expression, as described in Sec. 5. The extra $y \mapsto y^{\prime}$ variable substitutions we must perform get lumped into the substitution underneath the copied $\lambda$-expression (but are not needed above the copied $\lambda$-expression). Again, the substitutions are passed around the functions primarily as bookkeeping device that allows us to assert the necessary invariants at different points in the function definition. CG is an invariant across both Sub and uc.

- $\operatorname{CMS}[g]$

"Cache mirrors structure." That is, new graph structure "mirrors" old graph structure. If two connected nodes $n \stackrel{\mathrm{lbl}}{\longrightarrow} m$ are both in the domain of the cache, then their images in the cache are connected by the same kind of edge: $(g . \rho n) \stackrel{\text { lbl }}{\longrightarrow}(g . \rho m)$. This means that if we start at a node in $g_{0}$ and trace out a path downwards, always confining ourselves to nodes in the domain of the current cache, then the image of 


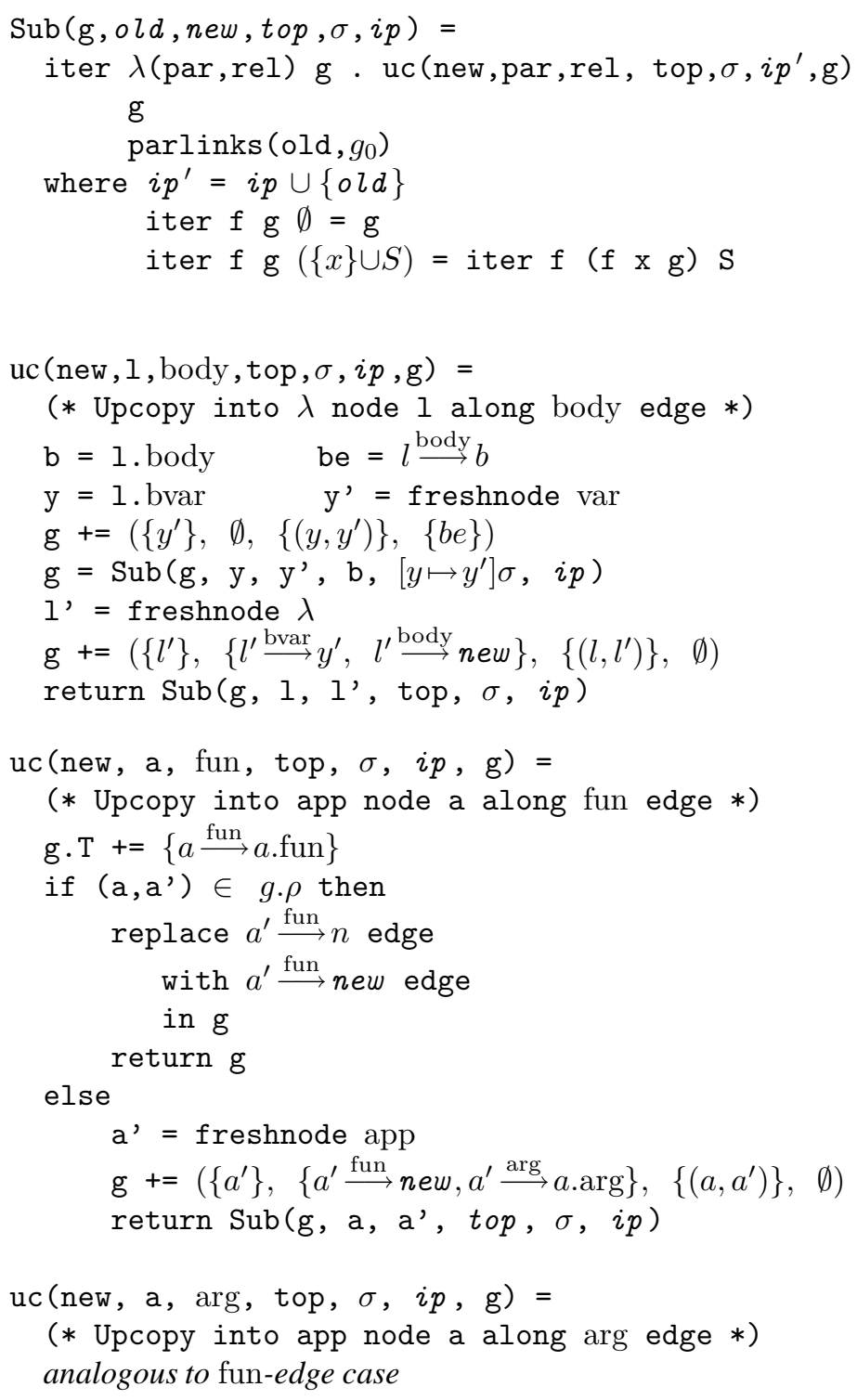

Figure 5: The core substitution algorithm, in abstract form. 
this path in the cache traces out an identical path through new structure in $g$. CMS is an invariant across both Sub and uc.

- $C P E T[g, i p]$

"Cached-node parent edges traced." If we have visited (hence cached) a node $n$, then all parent edges $p \rightarrow n$ have been traced (that is, are in $g . T$ ), unless (1) $n$ is currently "in-process $(n \in i p)$, or (2) $n=t_{o p}$, the top value passed to the initial Sub call. CPET is an invariant across both Sub and uc. The ip set is how we handle the fact that an upcopy triggered by a $\lambda$ cascade can terminate on an in-process node, who is in the cache, but whose processing is currently unfinished.

- $T P C[g, i p]$

"Traced parents cached." Furthermore, if we have traced up through a $p \rightarrow n$ edge (that is, if the edge is in the graph's traced set $g . T$ ), then the parent $p$ is in the cache. $T P C$ is an invariant across both Sub and uc.

- $A N V[g, t, b, i p]$

"All nodes visited." Any node $n$ on a path $t \longrightarrow^{*} b$ from top node $t$ to bottom node $b$ in $g_{0}$ must be in the cache, where we require that the $n \longrightarrow^{*} b$ suffix of the path be ipfree. (Note that when ip is empty, this simply says that all nodes on all paths from $t$ to $b$ are in the cache.) $A N V[g, t o p, o l d, i p]$ and $A N V[g, t o p, p a r, i p]$ are post-conditions of Sub and uc, respectively.

- Monotonicity

For both Sub and uc, all components of the input graph are preserved in the output graph, except for the edge set: $g . N \subset g^{\prime} . N \wedge g . \rho \subset g^{\prime} . \rho \wedge g . T \subset g^{\prime} . T$, where $g^{\prime}$ is the output graph. Also, we have the invariant that the graph always contains the original graph: $g_{0} \subset g$.

These are not all the necessary pre- and post-conditions, but they are the major ones.

The main "entry point" for the reduction algorithm is the $\operatorname{Sub}(g$, old, new, top, $\sigma$, ip $)$ function, which performs an $[$ old $\mapsto n e w]$ substitution in $g$, returning the augmented graph. Consider the initial call to Sub, in response to a desire to reduce some redex $(\lambda x . n) m$. Let $\widehat{m}$ be the unfolded $\Lambda_{\text {tree }}$ term for $m$. Assume that top is the topmost application under $n$-if there is no such node, then simple special-cases apply, as discussed in Sec. 5. Let

$$
\begin{aligned}
\text { top }^{\prime} & =\text { a fresh node not in } g_{0}, \\
\rho^{\prime} & =\rho \cup\left\{(x, m),\left(\text { top }, t^{\prime} p^{\prime}\right)\right\}, \text { and } \\
e^{\prime} & =\left\{\text { top }^{\prime} \stackrel{\text { fun }}{\longrightarrow} \text { top.fun, } \text { top }^{\prime} \stackrel{\text { arg }}{\longrightarrow} \text { top.arg }\right\} \\
g_{1} & =g_{0} \cup\left(\left\{\text { top }^{\prime}\right\}, e^{\prime}, \rho^{\prime}, \emptyset\right) .
\end{aligned}
$$

Then $\operatorname{Sub}\left(g_{1}, x, m\right.$, top, $\left.[x \mapsto \widehat{m}], \emptyset\right)$ satisfies the preconditions.

To see the rough picture of how the post-conditions give us the proper substitution, consider that after Sub returns, $A N V$ tells us that all nodes on the paths between top and $x$ are in the cache; this, together with the "cache mirrors structure" post-condition and the fact that $g . \rho x=m$, tells us the new term is what we wanted.

Sub is a fairly simple function; it just uses the uc function to initiate old $\mapsto n e w$ upcopies through every link from old up to some parent. The presence of top in the cache causes all of these upcopies to terminate; the new graph structure they create is entered into the cache. While the upcopies are being performed, old is kept in the in-process set $i p$.

The uc function is defined in Fig. 5 in three cases: upcopying into a $\lambda$-expression, upcopying through a fun link into an application node, and upcopying through an arg link into an application node (this last case is not given; it is entirely similar to the arg-link case). Note how, in the $\lambda$ case, the pre-Sub code sets up the preconditions for the first Sub call, which triggers the $y \mapsto y^{\prime}$ variable-substitution cascade-in particular, the $\left[y \mapsto y^{\prime}\right]$ mapping 
must be added to the prediction substitution $\sigma$. This step sets up the pre-conditions for the upward continuation of the algorithm, which substitutes $\lambda y^{\prime}$.new for $\lambda y$.old, completing the upcopy.

\section{Experiments}

To gain experience with the algorithm, a pair of Georgia Tech undergraduates implemented three $\beta$-reduction algorithms in SML: the bottom-up algorithm (BUBS), a reducer based on the suspension $\lambda$ calculus (SLC, see Sec. 10.1), and a simple, base-line reducer, based on the simple top-down, blind-search recursive procedure described in Sec. 1. They also built a tool-chain that allowed us to convert between a concrete s-expression form for terms and the particular representations required for the various algorithms. This allowed us to test the algorithms by running all three on randomly constructed trees, and comparing the results for discrepancies.

We then implemented two normalisers for each algorithm, one evaluating to normal form; the other, to weak-head normal form. The normalisers are called with an integer "reduction budget"- after performing that many reductions, they give up.

We first ran a few tests designed to show the bottom-up algorithm at its best and worst. The first test reduced a "chain of pearls" stack of applications, 20 deep, with full sharing and the identity combinator $I=\lambda x . x$ at the leaf:

$$
\begin{gathered}
@ \\
(@) \\
\vdots \\
@ \\
(1) \\
\text { I }
\end{gathered}
$$

This set up the bottom-up algorithm to do very well, since it can exploit the sharing to achieve exponential speedup. (Note, in particular, that the bottom-up algorithm is what the graph-reduction community calls "fully lazy." That is, reductions on dynamically-created shared structure are seen by all parents of that structure.)

Second, we normalised a 40,000-long chain of the form $(\lambda y . \lambda x . \lambda x \ldots \lambda x . y) \lambda z . z$. This is an example where even the naïve algorithm can do well, as there is no search involved - the branch factor of a chain of nested $\lambda$-expressions is one. So this shows off the "constant factors" of the algorithm.

Third, we normalised a full binary tree, 20 deep, of applications, with identity combinators at the leaves. This is the same term as the one in the first test, but with no sharing of structure. This is designed to show the algorithm at its worst, since there is no sharing at all during the entire normalisation process.

All normalisations were executed with a budget of 1,000 reductions. Here are the timings from these runs:

\begin{tabular}{|l|lll|}
\hline & 20 pearls & $40 \mathrm{k} \lambda$ & tree20 \\
\hline BU & $0+0$ & $15+0$ & $9+84$ \\
BU-nocheck & $0+0$ & $318+1433$ & $10+80$ \\
SLC & N/A & $70+73$ & $16+72$ \\
Simple & N/A & $364+1536$ & $1+3$ \\
\hline
\end{tabular}

The "BU-nocheck" entries are for the bottom-up algorithm with the fast-path single-parent optimisation (described in Sec. 6) turned off. The tests were run using SML/NJ 110.42 on a 667Mhz G4 Powerbook with 512MB of PC133 RAM under Mac OS X 10.2.2. The 20length chain-of-pearls entry could not be run for SLC or the simple algorithm, as it requires a representation that can handle DAGs; the numbers for these algorithms are essentially those for the equivalent, unfolded 20-deep full application tree in the third column. Each pair of numbers $e+g$ is the time in milliseconds $e$ to execute the algorithm, and the time $g$ spent in garbage collection. Note that the bottom-up algorithm is able to polish off the 


\begin{tabular}{|l||r|r|r||r|r||}
\cline { 2 - 6 } \multicolumn{1}{c||}{} & \multicolumn{3}{c||}{ CPU time $(\mathrm{ms})$} & \multicolumn{2}{c||}{ \# reductions } \\
\cline { 2 - 6 } \multicolumn{1}{c||}{} & BUBS & SLC & Simple & BUBS & Tree \\
\hline (fact 2) & 0 & 10 & 10 & 123 & 180 \\
fact 3) & 0 & 20 & 20 & 188 & 388 \\
\hline (fact 4) & 0 & 40 & $\infty$ & 286 & 827 \\
(fact 5) & 0 & 160 & $\infty$ & 509 & 2045 \\
\hline (fact 6) & 10 & 860 & $\infty$ & 1439 & 7082 \\
(fact 7) & 20 & 5620 & $\infty$ & 7300 & 36180 \\
\hline (fact 8) & 190 & 48600 & $\infty$ & 52772 & 245469 \\
nasty-I & 30 & 740 & $\infty$ & 7300 & 8664 \\
\hline pearl10 & 0 & N/A & N/A & 10 & N/A \\
pearl18 & 0 & N/A & N/A & 18 & N/A \\
\hline tree10 & 0 & 0 & 0 & 1023 & 1023 \\
tree18 & 740 & 2530 & 1980 & 262143 & 262143 \\
\hline
\end{tabular}

Figure 6: Timings for $\mathrm{C}$ implementations.

40,000-element chain of nested $\lambda$-expressions easily, as this can be handled by the singleparent fast path in constant time.

Experience with these runs, and others, led us to implement a tightly coded $\mathrm{C}$ implementation of the same three algorithms to get more accurate measurements. The SML version had several issues that affected measurement. The implementation contains some redundant safety tests that the type system is unable to eliminate. SML also limits our ability to do detailed layout of the data structures. For example, by allocating the uplink structure in the same block of storage as the parent node to which it refers, we can move from the uplink to the referenced parent node with pointer arithmetic (i.e., on-processor), instead of needing to do a slower memory load. Similarly, if we allocate a $\lambda$-expression and its bound variable together, in a single memory block, we can move between these two structures without doing memory traffic. Finally, $\mathrm{C}$ eliminated our dependence on the garbage collector. The bottom-up algorithm intrinsically does its own storage management, so we don't need a GC. We get a fair amount of GC overhead in our SML implementation, $\mathrm{SML} / \mathrm{NJ}$, as it allocates even procedure call frames in the heap.

The SLC and simple reducers written in C managed storage with the Boehm-DemersWeiser garbage collector, version 6.2. We compiled the code with gcc 2.95.4 -g -O2 -Wall and performed the test runs on an $800 \mathrm{MHz}$ PIII (256 KB cache), $128 \mathrm{MB}$ RAM, Debian GNU/Linux 3.0 system. The timings for these runs are shown in Fig. 6. The system gave us a measurement precision of $10 \mathrm{~ms}$; an entry of $0 \mathrm{~ms}$ means below the resolution of the timer-i.e., less than $10 \mathrm{~ms}$; a measurement of $\infty$ means the measurement was halted at 10 cpu-minutes (or, in one case, at $2 \mathrm{~min} 20 \mathrm{sec}$ due to severe page thrashing).

Fact entries are factorial terms, with Church-numeral encodings. Nasty-I is a complex, hand-generated, tree of $\mathrm{K}$ and $\mathrm{S}$ combinators that reduces to I; the tree contains 20,152 nodes. The "pearl $i$ " and "tree $i$ " terms are as described for the SML timings.

We caution the reader from drawing too much from these timings. They are fairly preliminary. We are undertaking to perform a larger series of tests, in order to get a better understanding of the algorithm's performance.

We also do not wish to claim that the bottom-up algorithm is a competitive standalone graph-reduction system. Modern graph-reducers are highly-engineered systems that employ a battery of static analyses and run-time optimisations to gain performance. The bottom-up reducer, in contrast, embodies a single idea. Perhaps this idea could be applied to the production of a competitive graph-reducer; we chose term normalisation simply as a generic task that would thoroughly exercise the reduction engines. We are also quite willing to believe that a complex algorithm such as the read phase of the suspension $\lambda$ calculus has 
opportunities for clever speedups that our simple implementation did not implement. Further, the experiments we performed do not show the SLC algorithm at its best-it is specifically tuned to provide an extra capability that, for applications such as theorem provers, can provide tremendous "application-level" speedups: laziness. Normalisation examines the entire final term, thus eliminating some of the benefit of SLC's laziness.

That all said, the bottom-up algorithm is obviously very fast. Part of this speed comes from "full laziness:" a reduction of a shared redex is shared by all the parents of the redex. But this is not the whole story-the (fact 8 ) case does $1 / 5$ of the reductions, but gets a speedup of $256 \mathrm{x}$, and the tree 18 case has no sharing at all, but still manages a speedup of $3.4 x$ (over SLC, that is; the speedups over the simple reducer are different, but the general picture is similar). This is primarily due to the elimination of blind search, and consequent ability to share structure across a reduction step (as opposed to within a term).

One of the striking characteristics of the bottom-up algorithm is not only how fast it is, but how well-behaved it seems to be. The other algorithms we've tried have fast cases, but also other cases that cause them to blow up fairly badly. The bottom-up algorithm reliably turns in good numbers. We conjecture this is the benefit of being able to exploit both sharing and non-sharing as they arise in the DAG. If there's sharing, we benefit from re-using work. If there's no sharing, we can exploit the single-parent fast path. These complementary techniques may combine to help protect the algorithm from being susceptible to particular inputs.

\section{Related work}

A tremendous amount of prior work has been carried out exploring different ways to implement $\beta$-reduction efficiently. In large part, this is due to $\beta$-reduction lying at the heart of the graph-reduction engines that are used to execute lazy functional languages. The text by Peyton Jones et al. [13] summarises this whole area very well.

However, the focus of the lazy-language community is on representations tuned for execution, and the technology they have developed is cleverly specialised to serve this need. This means, for example, that it's fair game to fix on a particular reduction order. For example, graph reducers that overwrite nodes rely on their normalisation order to keep the necessary indirection nodes from stacking up pathologically. A compiler, in contrast, is a $\lambda$-calculus client that makes reductions in a less predictable order, as analyses reveal opportunities for transformation.

Also, an implementation tuned for execution has license to encode terms, or parts of terms, in a form not available for examination, but, rather, purely for execution. This is precisely what the technique of supercombinator compilation does. Our primary interest at the beginning of this whole effort was instead to work in a setting where the term being reduced is always directly available for examination-again, serving the needs of a compiler, which wants to manipulate and examine terms, not execute them.

\subsection{Explicit-substitution calculi}

One approach to constructing efficient $\lambda$-term manipulators is to shift to a language that syntactically encodes environments. The "suspension $\lambda$ calculus" developed by Nadathur et al. [12] is one such example that has been used with success in theorem provers and compilers. Being able to syntactically represent environments allow us to syntactically encode term/environment pairs to represent closures. This means $\beta$-reduction can be done in constant time, by simply producing a closure over the $\lambda$-expression's body in an environment mapping its variable to the redex's argument term.

The point of doing so is laziness, of two particular forms. First, we can reduce a closure term incrementally, doing only enough work to resolve whether its top-level is a variable reference, an application or a $\lambda$-expression, while leaving any child terms (such as the body of a $\lambda$-expression) suspended, i.e., explicit closures. Thus the work of completely resolving 
a closure created by a reduction into a tree composed of simple variable/application/ $\lambda$ expression terms can be done on a pay-as-you-go basis. For example, a theorem prover that wishes to compare two terms for equality can recursively explore the terms, resolving on the fly, but abandon the recursive comparison as soon as two subterms fail to match. By lazily resolving the tree, no work is done to resolve parts that are not needed.

Second, the reduction system for the expanded language includes rules for merging two substitution environments together before applying the single compound substitution to a term. This converts a double pass over the term into a single pass.

The great payoff for using a term-manipulation engine based on the SLC comes for systems that can exploit laziness. A program that examines the entire tree produced by a given reduction, however, is not going to benefit as much from the laziness. A compiler, for example, is a program that typically "walks" the entire program structure given to it, performing analyses and transforms on the structure.

SLC reduction, in the terms we've defined, uses "blind search" to find the variables being substituted. This cost is mitigated by its ability to merge environments-it increases the odds that searching down a particular link will turn up some variable that needs to be replaced. On the other hand, this is, to some degree, just shifting work back to the environment-merging calculations, which are not trivial.

The SLC also has strong barriers to sharing internal structure as a DAG. This is a consequence of its representation of terms using de Bruijn indices, which are context-dependent: a term with free variable references will have two distinct forms at two different places in the tree. Again, this is somewhat mitigated by the SLC's ability to place a term in an environment and then produce a simple closure (which might contain multiple references to the bound term) with that environment. However, by the time a final, base term has been completely produced, all environments must be removed, and so the replication has to be performed at some point.

On the other hand, if SLC terms are not resolved but instead left suspended, then a different space issue arises: redundantly bound and unreferenced variable bindings can persist in suspensions, causing space leaks. Eliminating these leaks requires trimming environments, which is a time cost of its own.

The uplinked $\lambda$-DAG representation explicitly provides for sharing, and does so in a way that allows clients of the data structure to operate on the entire structure without having to unfold it into its equivalent tree, an operation that could induce exponential blowup in space. The issue of leaks due to unreferenced or redundant bindings does not arise at all.

Finally, the SLC is a quite sophisticated algorithm. The fine details of its reduction rules are fairly subtle and non-obvious. It requires transforming the term structure into a related, but very distinct form: nameless terms, with new and complex non-terminals expressing suspended reductions and their environments, in various states of merging.

SLC has been successfully employed inside a compiler to represent Shao's FLINT typed intermediate language, but the report on this work [15] makes clear the impressive, if not heroic, degree of engineering required to exploit this technology for compiler internalsthe path to good performance couples the core SLC representation with hash consing as well as memoisation of term reductions.

The charm of the bottom-up technique presented here is its simplicity. The data structure is essentially just a simple description of the basic syntax as a datatype, with the single addition of child $\rightarrow$ parent backpointers. It generalises easily to the richer languages used by real compilers and other language-manipulation systems. It's very simple to examine this data structure during processing; very easy to debug the reduction engine itself. In contrast to more sophisticated and complex representations such as SLC, there are really only two important invariants on the structure: (1) all variables are in scope (any path upwards from a variable reference to the root must go through the variable's binding $\lambda$-expression), and (2) uplink backpointers mirror downlink references. 


\subsection{Director strings}

Director strings [7] are a representation driven by the same core issue that motivates our uplinked-DAG representation: they provide a way to guide search when we perform a $\beta$ reduction. In the case of director strings, however, one can do the search top-down.

At each application node, the term's free variables are sorted by lexical height. Then, each application node is annotated with a string of symbols drawn from the set $\{/, \backslash, \wedge, 0\}$, one symbol for each free variable. The symbol used for a given variable tells if the variable occurs in the left child only, the right child only, both children, or neither child, respectively. (The 0 symbol is only used in degenerate cases.) These strings provide the information needed to do top-down guided search, in a fashion similar to the binary-tree insertion algorithm of Sec. 2 .

Director strings, however, can impose a quadratic space penalty on our trees. The standard example showing this is the term $\lambda x_{1} \ldots \lambda x_{n} .\left(x_{n} \ldots x_{1}\right)$. Uplinked $\lambda$-DAGs are guaranteed to have linear space requirements. Whether or not the space requirements for a director-strings representation will blow up in practice depends, of course, on the terms being manipulated. But the attraction of a linear-space representation is knowing that blowup is completely impossible.

Like the suspension $\lambda$ calculus, director strings have the disadvantage of not being a direct representation of the original term; there is some translation involved in converting a $\lambda$-calculus term into a director-strings form.

Director strings can be an excellent representation choice for graph-reducing normalising engines. Again, we are instead primarily focussed on applications that require finegrained inter-reduction access to the term structure, such as compilers.

\subsection{Optimal $\lambda$ reduction}

The theory of "optimal $\lambda$ reduction" $[10,9,6]$ (or, OLR), originated by Lévy and Lamping, and developed by Abadi, Asperti, Gonthier, Guerrini, Lawall, Mairson et al., is a body of work that shares much with bottom-up $\beta$-reduction. Both represent $\lambda$-terms using graph structure, and the key idea of connecting variable-binders directly to value-consumers of the bound variable is present in both frameworks - and for the same reason, namely, from a desire that substitution should be proportional to the number of references to the bound variable, removing the need to blindly search a term looking for these references.

However, the two systems are quite different in their details, in fairly deep ways. The "Lamping graphs" of optimal $\lambda$ reduction add extra structure to the graph, in the form of "croissants," "brackets," and "fan" nodes, to allow a novel capability: incremental $\beta$ reduction. Reduction is not an atomic operation in OLR. It can be performed in multiple steps; intermediate stages of the reduction are valid graphs. (The croissant and bracket marks delimit the boundaries of a reduction step as they propagate through the graph.) This is an enormous difference with our simple bottom-up $\beta$-reduction system-it is, essentially, an exciting and different model of computation from the one based on the classical $\lambda$ calculus. However, it comes with a cost: the greatly increased complexity of the graph structure and its associated operations. As Gonthier, Abadi and Lévy state [6], "it seems fair to say that Lamping's algorithm is rather complicated and obscure."

The details of this complexity have prevented OLR-based systems from being used in practice. We note that it has been fourteen years since the original innovation of Lamping graphs, and no compiler or theorem prover has adopted the technology, despite the lure of guaranteed optimality and the very real need [15] of these systems for efficient representations. (The OLR researchers themselves have implemented a graph-reduction engine using the OLR algorithm, but, as we've stated, this application is not our main concern, here.) In particular, in actual use, the croissant and bracket marks can frequently pile up uselessly along an edge, tying up storage and processing steps. It also makes it difficult to "read" information from the graph structure. In this respect, OLR remains the province of theoreticians, not implementors. 
Optimal $\lambda$ reduction comes with a great deal of theoretical underpinnings. Of particular note is that it makes a claim to optimality, in terms of using sharing to guarantee the minimal number of reductions. We make no such claim; it is clear that the bottom-up algorithm is not optimal, in the narrow technical sense that it will replicate some $\lambda$-terms that would not be replicated by an OLR reducer. Again, the OLR reducer would achieve this sharing by inserting fan, bracket and croissant nodes into the graph-greatly complicating the graph structure and readback problem. Asperti and Guerrini's comprehensive text [1] devotes fifty pages to the topic of readback; it is not a trivial issue. Further, the accumulation of croissant and bracket nodes during reduction is not currently well understood or characterised, so OLR's optimality of reductions must be offset by this accompanying cost.

OLR work may yet well lead to the development of practical reduction techniques that exploit the sharing of Lamping graphs, but we are not there yet. A weaker engineering goal is to relax notions of sharing, and develop functionally correct algorithms that still have good performance behavior, more in the pragmatic style of the classic graph-reduction community $[13,17]$. Our research is better appreciated in that context.

\subsection{Two key issues: persistence and readback}

Our comparisons with other techniques have repeatedly invoked the key issues of persistence and readback. Our data structure is not a "persistent" one-performing a reduction inside a term changes the term. If an application needs to keep the old term around, then our algorithm is not a candidate (or, at least, not without some serious surgery). So perhaps it is unfair to compare our algorithm's run times to those of persistent algorithms, such as SLC or director strings.

However, we can turn this around, and claim that the interesting feature of our algorithm is that it exploits lack of persistence. Applications that need persistence are rare in practice - and if an application doesn't need persistence, it shouldn't have to pay for it. The standard set of technology choices are invariably persistent; our algorithm provides an alternative design point. (Note that reduction on Lamping graphs is also not persistent, which is, again, either a limitation or a source of efficiency, depending on your point of view.)

The other key, cross-cutting issue is readback. An application that doesn't need to examine term structure in-between reductions has greater flexibility in its requirements. If readback is a requirement, however, then Lamping graphs and the SLC are much less attractive. Readback with our representation is free: one of the pleasant properties of a DAG is that it can be viewed just as easily as a tree; there is no need to convert it.

Thus, bottom-up $\beta$-reduction is a technology which is well suited to applications which (1) don't need persistence, but (2) do need fine-grained readback.

\section{Other operations: cloning, equality and hashing}

$\beta$-reduction is, of course, not the only operation one might wish to perform on terms of the $\lambda$ calculus. In a DAG representation, we also might wish to provide a parent-splitting operation, to unshare a node that has multiple parents. This means cloning the child node, and dividing the parents between the original child and its copy as indicated by some partition. This operation, which is easy to implement, would be useful for a compiler that made reduction (aka "inlining") decisions based on context. If we want to contract a redex in one context, but leave it as-is in another, we must first replicate the node, so the two contexts have distinct redexes. Note that only the top of the redex needs to be replicated; the two redex copies can share children, so this operation is fast. (Cloning a $\lambda$-expression is more work than cloning an application, however: the $\lambda$ 's bound variable must also be replicated to preserve $\alpha$-uniqueness. This kicks off an up-copy along the paths from the variable up to the $\lambda$-expression being cloned, just as in the $\beta$-reduction case. However, applications, not $\lambda$-expressions, are the nodes one typically wishes to clone in order to perform contextsensitive reductions.) 
Comparing terms for equality brings up the question of which definition of equality we mean. The spectrum runs from complete textual equality, to tree equality modulo $\alpha$-conversion, to extensional equality in the model of a denotational semantics. The presence of sharing in our DAG representation raises new distinctions, as well. One useful definition of equality for DAGs is: if we expanded term DAGs $t_{1}$ and $t_{2}$ into their equivalent trees, would these trees be structurally equal, that is, equal modulo $\alpha$-conversion? Implementing this efficiently is a nice puzzle. Note that we can't use the usual trick of converting to de Bruijn indices and comparing the results-the DAG representation completely rules out the use of de Bruijn indices, as there may be two paths from a variable up to its binding $\lambda$-expression that run through different numbers of intermediate $\lambda$-expressions!

We have designed and implemented an algorithm for this equality test that is "almost linear" (in the sense of the inverse Ackermann function) in the sizes of the DAGs; the algorithm uses the fast amortised union-find algorithm for its speed. A detailed discussion of the $\alpha$-DAG equality algorithm is beyond the scope of this article; we expect to describe it fully in another report.

It is also important for many potential uses of $\lambda$-terms to have a hash function that is insensitive to sharing and $\alpha$-conversion, i.e., one that respects the equality test outlined above. Such a facility has, in fact, been universally requested by colleagues who are beginning to use our technology for their own projects. In particular, it enables "hash cons" construction of $\lambda$-terms, reducing the cost of $\alpha$-equivalence tests to a single pointer comparison. A further criteria for a good hash function is that it should be "incrementally" computable, that is, we would like to be able to compute efficiently the hash value for a $\lambda$ or application node from the hash values for its children. Similar considerations of incrementality apply to rehashing term structure as needed across a $\beta$-reduction.

We have designed and implemented three different hash functions for bubs terms, and are currently engaged in evaluating them in support of hash-consing terms in a compiler based on a three-level/kinded typed-intermediate language. We should note that, despite our colleague's entreaties, it is not a given that hash-consing will provide much improvement in our setting. Hash-consing provides a fast-path for $\alpha$-equality, but our equality function is already fairly fast. When we say that it is almost linear, we mean almost linear in the size of the DAGs, not their unfolded trees, so the structure-sharing enabled by the representation can potentially provide tremendous speedups - and it is only linear when the terms turn out to be equal; it can quit lazily as soon as it encounters a structural difference between the terms. Hash consing also provides for space savings due to sharing; again, our basic representation already picks up sharing that occurs due to reduction. We await tests on real data to see how things will measure.

\section{Possible variants and applications}

\subsection{Cyclic graph structure}

It would be interesting to see if the algorithm could be adapted to operate on general graph structure, as opposed to DAGs. This would permit recursion to be captured with circular structure, as opposed to encoding it using syntactic devices such as the $\mathrm{Y}$ combinator.

The basic marking-based search\&copy technique is one that works on general graphs with no trouble. However, one complication in such a framework is that a reduction that unrolls a recursion can cause uplinks to become downlinks. This perturbs some of the fundamental invariants on which the algorithm is based, and so affects much of the code. Altering the algorithm to properly account for this behavior would require careful thought.

\subsection{Integrating with orthogonal implementation techniques}

As we've noted already, there is a tremendous body of work on the high-performance graph reduction of $\lambda$-calculus terms. The uplinked $\lambda$-DAG representation is not mutually exclusive with many of these techniques. It would be worth investigating to discover how much 
of the "classical" graph-reduction technology could be applied in this DAG framework. For example, could supercombinators be compiled into native code to operate on uplinked $\lambda$-DAG representations?

We have claimed in the past that every interesting programming language comes from an interesting model of computation. One of our colleagues has suggested the possibility that the model of computation embodied by the $\beta$-reduction of uplinked $\lambda$-DAGs might make for an interesting interpreted programming language.

\subsection{DAG-based compiler}

We are very interested in trying to put the bottom-up representation to use in a real compiler, to represent both program terms and sophisticated types. Type-based compilers [16, 14], in particular, are notorious for term explosion in the intermediate type terms; the sharing introduced by the bottom-up algorithm has potential to help here.

A compiler is an application that typically produces output proportional to the size of the intermediate code tree, which, in our case, is really the unfolded intermediate code tree. So perhaps there is less sharing payoff in representing program terms with a $\lambda$-DAG (as opposed to the type terms). However, this is only true of the final program term-program transforms and analyses could well benefit from sharing-based compression of the program term, avoiding term explosion in the intermediate stages. Further, even if the target language (i.e., assembler) doesn't allow sharing, we can still benefit from generating code from a DAG by caching the program-term-to-assembler translations. Finally, as we've described earlier, the bottom-up reduction algorithm gets time and space savings not only from sharing within a $\lambda$-term, but also from the elimination of blind search and the associated sharing of structure across a reduction-i.e., the reduction algorithm tries to copy as little graph structure as possible when reducing.

It is intriguing to consider what a compiler would be like that was more fundamentally based on representing program structure as a DAG (as opposed to using the bottom-up representation essentially as a short-hand for a tree). Costs and benefits are not always what they seem in this setting. For example, inlining a procedure definition by replacing the procedure's name with its $\lambda$-expression has no space cost in a DAG representation, even if the procedure is invoked at multiple call sites. Specialising such an application by subsequently contracting the $\lambda$-expression's application to a particular set of arguments is what causes code replication-although, even in this case, the bottom-up reduction algorithm attempts to share common structure.

\subsection{Graph-based compiler}

Even more intriguing and exotic is the possibility of allowing general graph structure for our compiler's internal structures. The question of variable scope (a tree notion), for example, becomes the more general question of binders dominating references (a graph-theoretic notion). We begin to verge, at this point, from the realm of $\lambda$ calculus to the realm of "flat" SSA representations [8]. The challenge is to do so, and yet retain the ideas - in their suitably generalised form-of scope and closure and higher-order functional values from the $\lambda$-calculus setting. At least one such compiler has been written, by Bawden [3], though the effort was never written up and published.

We do not claim that some sort of general-graph/circular-structure variant of the $\lambda$ calculus is a better way to build compilers. We do think it is an interesting idea to consider.

\section{Conclusion}

We certainly are not the first to consider using graph structure to represent terms of the $\lambda$ calculus; the ideas go back at least to $1954[4,17]$. The key point we are making is that two of these ideas work together:

- representing $\lambda$-terms as DAGS to allow sharing induced by $\beta$-reduction, and 
- introducing child $\rightarrow$ parent backpointers and $\lambda \rightarrow$ variable links to efficiently direct search and construction.

The first idea allows sharing within a term, while the second allows sharing across a reduction, but they are, in fact, mutually enabling: in order to exploit the backpointers, we need the DAG representation to allow us to build terms without having to replicate the subterm being substituted for the variable. This is the source of speed and space efficiency.

The algorithm is simple and directly represents the term without any obscuring transform, such as combinators, de Bruijn indices or suspensions, a pleasant feature for $\lambda$ calculus clients who need to examine the terms. It is also, in the parlance of the graphreduction community, fully lazy.

\section{Acknowledgements}

Bryan Kennedy and Stephen Strickland, undergraduates at Georgia Tech, did the entire implementation and evaluation reported in Sec. 9. Anonymous reviewers lent their expertise to the improvement of the paper. Zhong Shao provided helpful discussions on the suspension $\lambda$ calculus. Chris Okasaki and Simon Peyton Jones tutored us on director strings. Harry Mairson and Alan Bawden provided lengthy and patient instruction on the subtleties of optimal $\lambda$ reduction and Lamping graphs. Andrew Appel suggested to us the possibility of basing a programming-language semantics and implementation on the BUBS algorithm. Jean-Jacques Lévy also provided us with illuminating discussions on models of computation and the $\lambda$ calculus. We thank, of course, Olivier Danvy.

\section{References}

[1] Andrea Asperti and Stefano Guerrini. The Optimal Implementation of Functional Programming Languages. Cambridge University Press, 1999.

[2] Henk Barendregt. The Lambda Calculus. North Holland, revised edition, 1984.

[3] Alan Bawden. Personal communication, November 2002. Alan wrote the compiler, a toy exercise for Scheme, sometime in the late 1980's.

[4] N. Bourbaki. Théorie des ensembles. Hermann \& C. Editeurs, 1954.

[5] Alonzo Church. The Calculi of Lambda Conversion. Princeton University Press, 1941.

[6] Georges Gonthier, Martín Abadi and Jean-Jacques Lévy. The geometry of optimal lambda reduction. In Conference Record of the Nineteenth Annual ACM SIGPLANSIGACT Symposium on Principles of Programming Languages, pages 15-26, January 1992.

[7] J. R. Kennaway and M. R. Sleep. Director strings as combinators. ACM Transactions on Programming Languages and Systems, 10, pages 602-626, (October 1988).

[8] Richard A. Kelsey. A correspondence between continuation-passing style and static single assignment form. In ACM SIGPLAN Workshop on Intermediate Representations, SIGPLAN Notices, vol. 30, no. 3, pages 13-22, January 1995.

[9] John Lamping. An algorithm for optimal lambda-calculus reduction. In Proceedings of the Seventeenth Annual ACM Symposium on Principles of Programming Languages, pages 16-30, January 1990.

[10] Jean-Jacques Lévy. Réductions Correctes et Optimales dans le Lambda-calcul. Thèse d'État, Université de Paris VII, Paris, France, 1978. 
[11] R. Milner, M. Tofte, R. Harper, D. MacQueen. The Definition of Standard ML (Revised). MIT Press, 1997.

[12] Gopalan Nadathur and Debra Sue Wilson. A notation for lambda terms: A generalization of environments. Theoretical Computer Science 198(1-2):49-98, May 1998.

[13] Simon L. Peyton Jones. The Implementation of Functional Programming Languages. Prentice-Hall, 1987.

[14] Zhong Shao and Andrew W. Appel. A type-based compiler for Standard ML. In Proceedings of the ACM SIGPLAN'95 Conference on Programming Language Design and Implementation (PLDI), SIGPLAN Notices 30(6), pages 116-129, June 1995.

[15] Zhong Shao, Christopher League, and Stefan Monnier. Implementing typed intermediate languages. In Proceedings of the 1998 ACM SIGPLAN International Conference on Functional Programming Languages, September 1998.

[16] D. Tarditi, G. Morrisett, P. Cheng, C. Stone, R. Harper and P. Lee. TIL: A typedirected optimizing compiler for ML. In Proceedings of the ACM SIGPLAN'96 Conference on Programming Language Design and Implementation (PLDI), SIGPLAN Notices 31(5), pages 181-192, May 1996.

[17] C. P. Wadsworth. Semantics and pragmatics of the lambda-calculus. PhD dissertation, Oxford University, 1971. 


\section{A BetaSub.sml}

Not including comments, blank lines, or a simple doubly-linked list library, the source code for the core data structures and $\beta$-reduction algorithm is 180 lines of SML code; this includes the full set of optimisations discussed in Sections 5 and 6 . Here is a complete listing, liberally commented.

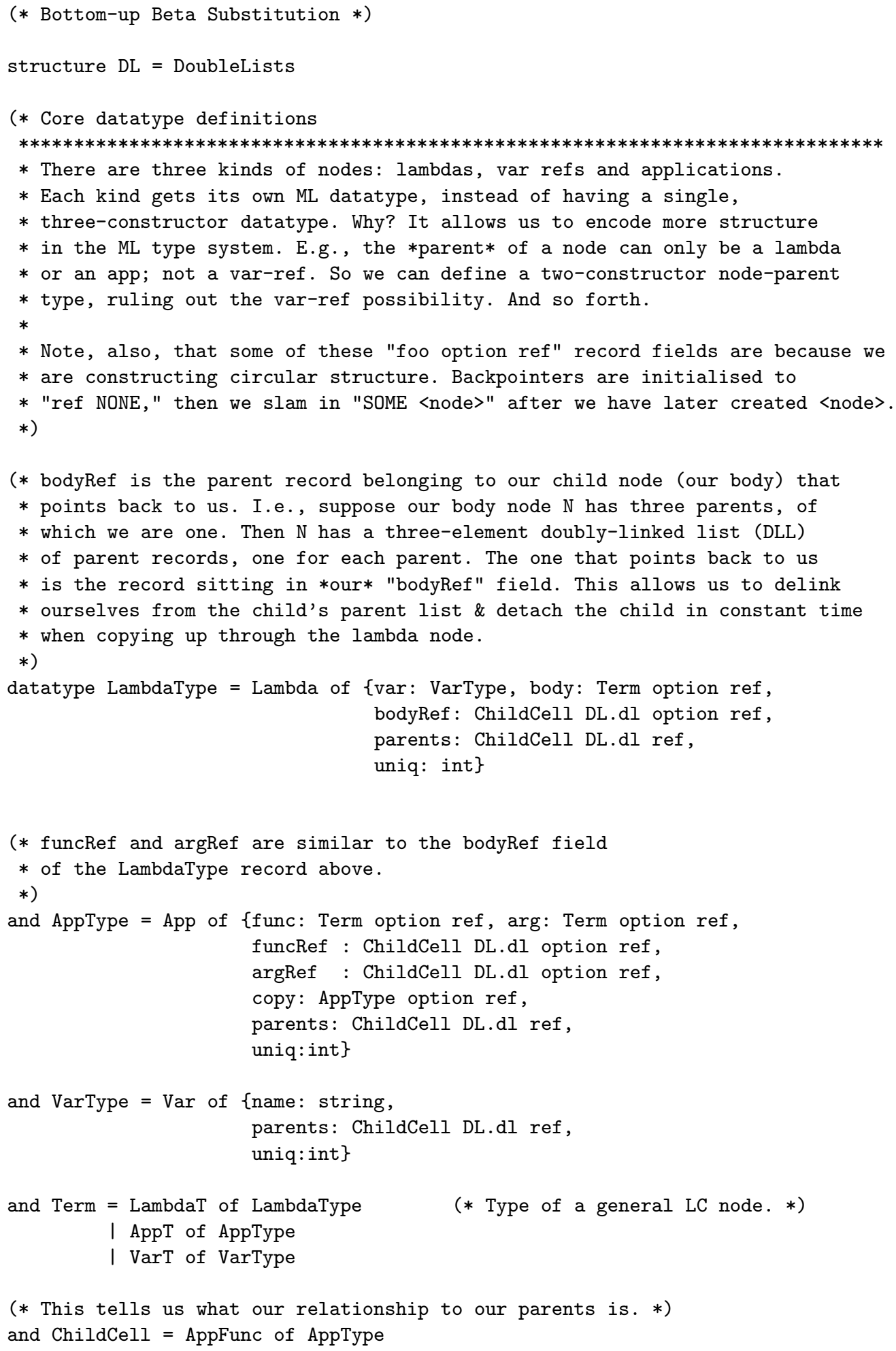


(* Get the parents of a Term. *)

fun termParRef (LambdaT (Lambda\{parents, ...\})) = parents

I termParRef(AppT(App\{parents, ...\})) = parents

I termParRef $(\operatorname{VarT}(\operatorname{Var}\{$ parents,$\ldots\})) \quad=$ parents

(* A rather subtle point:

$*$ When we do upsearch/copying, we chase uplinks/backpointers, copying old tree

* structure, creating new tree structure as we go. But we don't want to search

* up through *new* structure by accident -- that might induce an infinite

* search/copy. Now, the the only way we can have a link from an old node up to

* a new parent is by cloning an app node -- when we create a new app, it has

* one new child NC and one old child OC. So our new app node will be added to

* the parent list of the old child -- and if we should later copy up through

* the old child, OC, we'd copy up through the new app node -- that is, we'd

* copy the copy. This could get us into an infinite loop. (Consider reducing

* $(\backslash \mathrm{x} . \mathrm{x} \mathrm{x}) \mathrm{y}$

* for example. Infinite-loop city.)

* We eliminate this problem in the following way: we don't install *up* links

* to app nodes when we copy. We just make the downlinks from the new app node

* to its two children. So the upcopy search won't ever chase links from old

* structure up to new structure; it will only see old structure.

* We *do* install uplinks from a lambda's body to a newly created lambda node,

* but this link always goes from new structure up to new structure, so it will

* never affect the our search through old structure. The only way we can have a

* new parent with an old child is when the parent is an app node.

* When we are done, we then make a pass over the new structure, installing the

* func->app-node or arg->app-node uplinks. We do this in the copy-clearing

* pass -- as we wander the old app nodes, clearing their cache slots, we take

* the corresponding new app node and install backpointers from its children

* up to it.

* In other words, for app nodes, we only create downlinks, and later bring the * backpointer uplinks into sync with them.

*)

(* Given a term and a ChildCell, add the childcell to term's parents. *)

fun addToParents (node, cclink) = let val $\mathrm{p}=$ termParRef node in $\mathrm{p}:=$ DL.add_before(!p, cclink)

end

(* Is dll exactly one elt in length? *)

(* ML pattern matching rules.

fun len1 (DL.Node (_, , ref DL.NIL)) = true

I len1 _ = false

(* clearCopies (redlam, topapp)

$* * * * * * * * * * * * * * * * * * * * * * * * * * * * * * * * * * * * * * * * * * * * * * * * * * * * * * * * * * * * * * * * * * * * * * * * * * * * * * * *$

* When we're finished constructing the contractum, we must clean out the

* app nodes' copy slots (reset them to NONE) to reset everything for the next 
* reduction.

* - REDLAM is the lambda we reduced.

$*$

* - TOPAPP is the highest app node under the reduced lambda -- it holds

* the highest copy slot we have to clear out. If we clear it first, then

* we are guaranteed that any upwards copy-clearing search started below it

* will terminate upon finding an app w/an empty copy slot.

*

* Every lambda from REDLAM down to TOPAPP had its var as the origin of an

* upcopy:

* - For REDLAM, the upcopy mapped its var to the redex's argument term.

* - The other, intermediate lambdas *between* REDLAM \& TOPAPP (might be zero

* of these) were copied to fresh lambdas, so their vars were mapped to

* fresh vars, too.

* So, now, for each lambda, we must search upwards from the lambda's var,

* clearing cached copies at app nodes, stopping when we run into an

* already-cleared app node.

$*$

* This cache-clearing upsearch is performed by the internal proc cleanUp.

* (Get it?)

$*$

* When we created fresh app nodes during the upcopy phase, we *didn't*

* install uplinks from their children up to the app nodes -- this ensures

* the upcopy doesn't copy copies. So we do it now.

*)

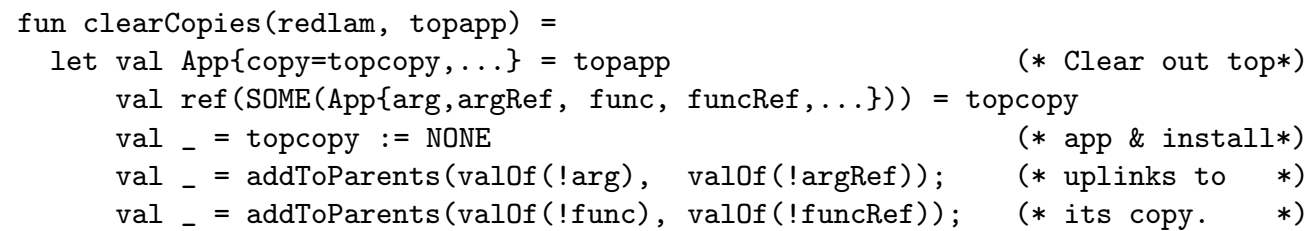

fun $\operatorname{cleanUp}(\operatorname{AppFunc}(\operatorname{App}\{c o p y=$ ref $\mathrm{NONE}, \ldots\}))=()$

I cleanUp (AppFunc(App\{copy as ref (SOME(App\{arg, argRef, func, funcRef,...\})),

(copy := NONE; parents, ... $)$ ) $=$

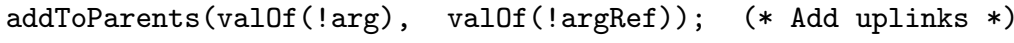
addToParents (valof (!func), valof (!funcRef)); (* to copy .

DL.app cleanUp (!parents))

| $\operatorname{cleanUp}(\operatorname{AppArg}(\operatorname{App}\{c o p y=r e f \quad \operatorname{NONE}, \ldots\}))=()$

I cleanUp(AppArg(App\{copy as $\operatorname{ref(SOME(App\{ arg,~argRef,~}$

(copy := NONE; parents, ...\})) = func, funcRef,...\})),

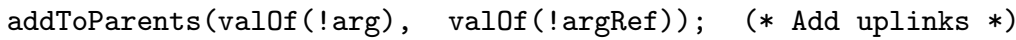
addToParents (valof (!func), valof (!funcRef)); (* to copy. *) DL.app cleanUp (!parents))

| $\operatorname{cleanUp}($ LambdaBody $($ Lambda\{parents, $\operatorname{var}, \ldots\}))=$ (varClean var; DL.app cleanUp (!parents))

and $\operatorname{varClean}(\operatorname{Var}\{$ parents=varpars,$\ldots\})=$ DL. app cleanUp (!varpars) 
fun lambdascan (Lambda\{var, body=ref (SOME b), .. $\})=$

(varClean var;

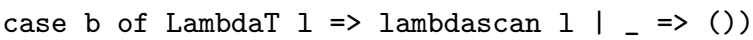

in lambdascan redlam

end

(* freeDeadNode term $\rightarrow$ unit

$* * * * * * * * * * * * * * * * * * * * * * * * * * * * * * * * * * * * * * * * * * * * * * * * * * * * * * * * * * * * * * * * * * * * * * * * * * * * * * * * *$

* Precondition: (termParents term) is empty -- term has no parents.

* A node with no parents can be freed. Furthermore, freeing a node

* means we can remove it from the parent list of its children... and

* should such a child thus become parentless, it, too can be freed.

* So we have a recursive/DAG-walking/ref-counting sort of GC algo here.

* IMPORTANT: In this SML implementation, we don't actually *do* anything

* with the freed nodes -- we don't, for instance, put them onto a free

* list for later re-allocation. We just drop them on the floor and let

* SML's GC collect them. But it doesn't matter -- this GC algo is *not

* optional*. We *must* (recursively) delink dead nodes. Why? Because

* we don't want subsequent up-copies to spend time copying up into dead

* node subtrees. So we remove them as soon as a beta-reduction makes

* them dead.

* So this procedure keeps the upwards back-pointer picture consistent with

* the "ground truth" down-pointer picture.

$*)$

fun freeDeadNode node $=$

let

fun free (AppT (App $\{$ func=ref (SOME functerm), funcRef, arg=ref (SOME argterm), argRef, parents, ...\})) =

(delPar (functerm, valOf(!funcRef)); (* Node no longer parent delPar(argterm, valof(!argRef $))$ ) (* of func or arg children.

$*)$

$*)$

I free(LambdaT (Lambda\{body=ref (SOME bodyterm), (* Lambda no longer $*$ ) delPar(bodyterm, valof(!bodyRef))

bodyRef, parents, ...\})) $=(*$ parent of body. *)

(* We wouldn't actually want to dealloc a parentless var node, because

* its binding lambda still retains a ref to it. Responsibility for

* freeing a var node should be given to the code (just above) that

* freed its lambda.

*)

I free $\left(\operatorname{Var} \mathrm{T}_{-}\right)=()$

(* Remove CCLINK from TERM's parent's dll.

* If TERM's parent list becomes empty, it's dead, too, so free it.

*)

and delPar(term, cclink) =

case DL.remove cclink of (* Returns the dll elts before \& after cclink. *)

(DL.NIL, after) $\Rightarrow$ let val parref $=$ termParRef term

in parref $:=$ after;

case after of DL.NIL $\Rightarrow$ free term

| DL.Node _ $\Rightarrow()$

$I_{-} \Rightarrow()$

end 
in free node

end

(* Replace one child w/another in the tree.

* - OLDPREF is the parent dll for some term -- the old term.

* - NEW is the replacement term.

* Add each element of the dll !OLDPREF to NEW's parent list. Each such

* element indicates some parental downlink; install NEW in the right slot

* of the indicated parent. When done, set OLDPREF $:=$ NIL.

$*$

* Actually, we don't move the dll elements over to NEW's parent list one at

* a time -- that involves redundant writes. E.g., if !OLDPREF is 23 elements

* long, don't move the elements over one at a time -- they are already nicely

* linked up. Just connect the last elt of !OLDPREF \& the first element of

* NEW's existing parent list, saving $22 * 2=44$ writes. Because it physically

* hurts to waste cycles.

*)

fun replaceChild(oldpref, new) =

let val cclinks $=$ !oldpref

val newparref = termParRef new

fun installChild(LambdaBody (Lambda\{body,...\})) = body := SOME new

| installChild(AppFunc (App\{func,...\})) = func := SOME new

| installChild(AppArg $(\operatorname{App}\{\arg , \ldots\})) \quad=\arg :=$ SOME new

fun $\operatorname{lp}($ prev, prevnext, DL.NIL $)=$

(prevnext := !newparref ;

case !newparref of DL.NIL $\quad \Rightarrow()$

| DL. Node $(p, \ldots, \ldots) \Rightarrow p:=$ prev $)$

| $\operatorname{lp}\left(\right.$ prev, prevnext, node as DL.Node $\left(\_, c c, n\right.$ as ref next)) = (installChild cc; lp (node, $\mathrm{n}$, next))

in case cclinks of DL.NIL $\Rightarrow$ ()

| node as DL.Node (_, cc,n as ref next) $\Rightarrow$

(oldpref := DL.NIL; installChild cc;

lp (node, $n$, next); newparref := cclinks)

end

(* Allocate a fresh lambda L and a fresh var V. Install BODY as the body of

* the lambda -- L points down to BODY, and L is added to BODY's parent list.

* The fresh var's name (semantically irrelevant, but handy for humans) is

* copied from oldvar's name.

$*$

* Once this is done, kick off an OLDVAR->V upcopy to fix up BODY should it

* contain any OLDVAR refs.

*)

fun newLambda (oldvar, body) =

let $\operatorname{val} \operatorname{Var}\{$ name, parents $=$ varparents, $\ldots\}=$ oldvar

val $\operatorname{var}=\operatorname{Var}\{$ name $=$ name,

uniq = newUniq () ,

parents $=$ ref DL.NIL

val bodyRefCell = ref NONE

val ans = Lambda\{var= var,

body $\quad=\operatorname{ref}($ SOME body),

bodyRef = bodyRefCell,

uniq = newUniq(), 
val cclink = DL.new (LambdaBody ans)

in bodyRefCell := SOME cclink;

addToParents (body, cclink);

(* Propagate the new var up through the lambda's body. *)

DL.app (upcopy (VarT var)) (!varparents);

LambdaT ans

end

(* Allocate a fresh app node, with the two given params as its children.

* DON'T install this node on the children's parent lists -- see "a subtle

* point" above for the reason this would get us into trouble.

$*)$

and newApp (func, arg) =

let val funcRef = ref NONE

val argRef = ref NONE

val app = App\{func = ref(SOME func),

$\arg \quad=\operatorname{ref}($ SOME $\arg )$,

funcRef $=$ funcRef,

argRef $=$ argRef,

copy = ref NONE,

parents $=$ ref DL.NIL,

uniq = newUniq ()$\}$

in funcRef $:=\operatorname{SOME}($ DL. new (AppFunc app) );

$\operatorname{argRef}:=\operatorname{SOME}(\mathrm{DL}$. new (AppArg app) );

app

end

(* upcopy newChild parRef $\rightarrow$ unit

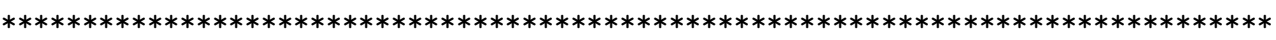

$*$ The core up-copy function.

* parRef represents a downlink dangling from some parent node.

* - If the parent node is a previously-copied app node, mutate the

* copy to connect it to newChild via the indicated downlink, and quit

* - If the parent is an app node that hasn't been copied yet, then

* make a copy of it, identical to parent except that the indicated downlink

* points to newChild. Stash the new copy away inside the parent. Then take

* the new copy and recursively upcopy it to all the parents of the parent.

* - If the parent is a lambda node L (and, hence, the downlink is the

* "body-of-a-lambda" connection), make a new lambda with newChild as

* its body and a fresh var for its var. Then kick off an upcopy from

* L's var's parents upwards, replacing L's var with the fresh var.

* (These upcopies will guaranteed terminate on a previously-replicated

* app node somewhere below L.) Then continue upwards, upcopying the fresh

* lambda to all the parents of L.

$*)$

and upcopy newChild (LambdaBody (Lambda\{var, parents, ... $\}$ )) =

DL.app (upcopy (newLambda (var, newChild))) (! parents)

(* Cloning an app from the func side *)

I upcopy new_child (AppFunc(App\{copy as ref NONE, arg, parents, ...\})) = let val new_app $=$ newApp (new_child, $\operatorname{val0f}(! \arg )$ )

in copy := SOME new_app;

DL.app (upcopy (AppT new_app)) (!parents) end

(* Copied up into an already-copied app node. Mutate the existing copy \& quit. *) 


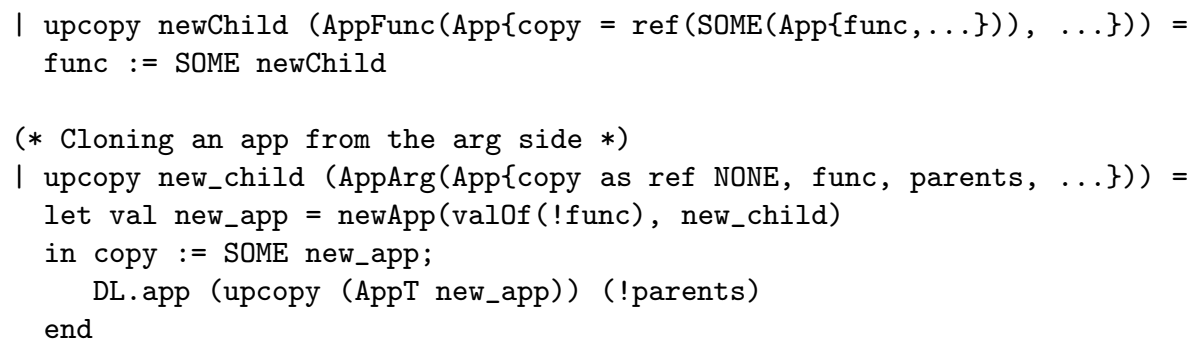

(* Contract a redex; raise an exception if the term isn't a redex. *)

fun reduce (a as $\operatorname{App}\{$ funcRef, func $=\operatorname{ref}(\operatorname{SOME}(\operatorname{LambdaT} 1))$, argRef, $\arg =\operatorname{ref}($ SOME argterm), parents, ...\}) $=$

let val Lambda $\{$ var, body, bodyRef, parents = lampars, $\ldots\}=1$

val $\operatorname{Var}\{$ parents $=$ vpars as ref varpars, $\ldots\}=$ var

val ans = if len1 (!lampars)

(* The lambda has only one parent -- the app node we're

* reducing, which is about to die. So we can mutate the

* lambda. Just alter all parents of the lambda's vars to

* point to ARGTERM instead of the var, and we're done!

$*)$

then (replaceChild(vpars, argterm); $\operatorname{val0f}($ ! body $))$

(* Fast path: If lambda's var has no refs,

* the answer is just the lambda's body, as-is.

$*)$

else if varpars = DL.NIL then valOf(! body)

(* The standard case. We know two things:

* 1. The lambda has multiple pars, so it will survive the reduction, and so its body be copied, not altered.

* 2. The var has refs, so we'll have to do some substitution.

* First, start at BODY, and recursively search down

* through as many lambdas as possible.

$*$

* - If we terminate on a var, the var is our lambda's var,

* for sure. (OTW, \#2 wouldn't be true.) So just return

* BODY back up through all these down-search lambda-

* skipping calls, copying the initial lambdas as we go.

* - If we terminate on an app, clone the app \& stick the

* clone in the app's copy slot. Now we can do our VAR->ARG

* up-copy stuff knowing that all upcopying will guaranteed

* terminate on a cached app node.

$*$

* When we return up through the initial-lambda-skipping

* recursion, we add on copies of the lambdas through

* which we are returning, *and* we also pass up that top

* app node we discovered. We will need it in the

* subsequent copy-clearing phase. 
$*)$

else let fun scandown ( $\mathrm{v}$ as $\left.\operatorname{VarT} \mathrm{T}_{-}\right)=(\operatorname{argterm}, \mathrm{NONE})(*$ No app! *)

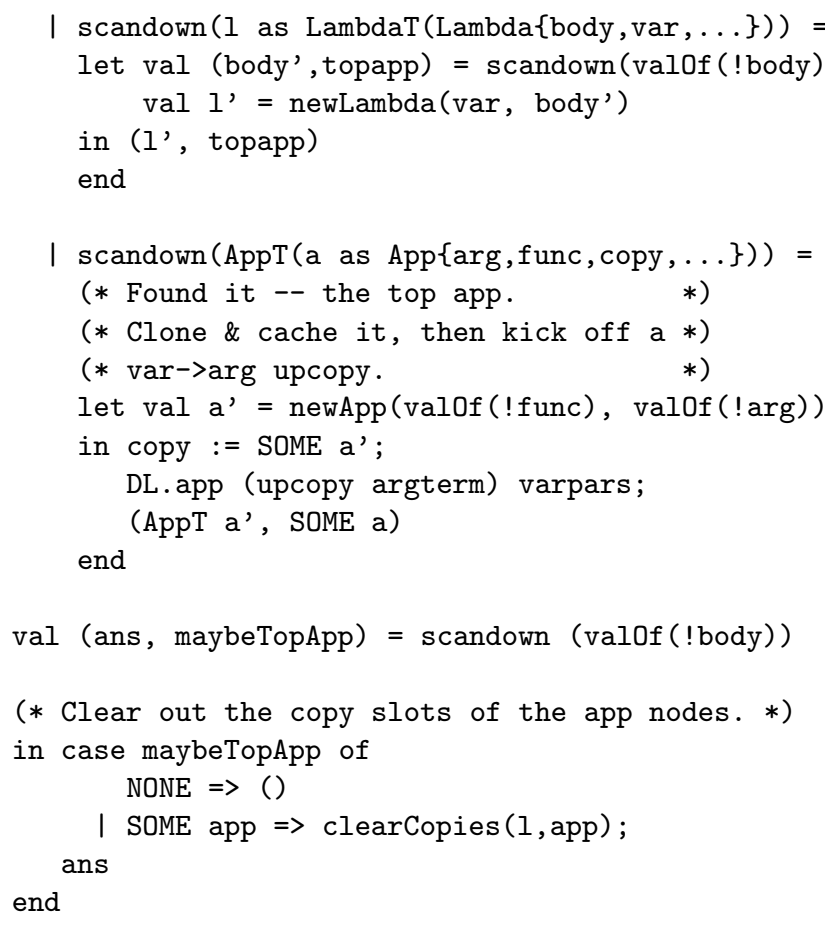

(* We've constructed the contractum \& reset all the copy slots. *)
in replaceChild(parents, ans); (* Replace redex w/the contractrum. *) freeDeadNode (AppT a); (* Dealloc the redex. *) ans
(* Done.

end

(* Call-by-name reduction to weak head-normal form. *)

fun normaliseWeakHead (AppT (app as App\{func, arg, $\ldots\}))=$ (normaliseWeakHead (valOf ( ! func)); case valOf(!func) of LambdaT _ $\Rightarrow$ normaliseWeakHead(reduce app)

I normaliseWeakHead _ $=()$ I $\quad \Rightarrow())$

(* Normal-order reduction to normal form. *)

fun normalise (AppT(app as App\{func, arg, uniq,...\})) = (normaliseWeakHead (valOf (!func));

case valof(!func) of LambdaT _ $\Rightarrow$ normalise (reduce app)

| VarT _ $\quad \Rightarrow$ normalise (valof (!arg))

| app' $\quad \Rightarrow$ (normalise app';

normalise $(\operatorname{val0f}((\arg ))))$

I normalise (LambdaT (Lambda\{body, ...\}) ) $=\operatorname{normalise}(\operatorname{val0f}($ ! body $))$

| normalise _ = () 


\section{Recent BRICS Report Series Publications}

RS-04-38 Olin Shivers and Mitchell Wand. Bottom-Up $\beta$-Substitution: Uplinks and $\lambda$-DAGs. December 2004. iv+32 pp.

RS-04-37 Jørgen Iversen and Peter D. Mosses. Constructive Action Semantics for Core ML. December 2004. 68 pp. To appear in a special Language Definitions and Tool Generation issue of the journal IEE Proceedings Software.

RS-04-36 Mark van den Brand, Jørgen Iversen, and Peter D. Mosses. An Action Environment. December 2004. 27 pp. Appears in Hedin and Van Wyk, editors, Fourth ACM SIGPLAN Workshop on Language Descriptions, Tools and Applications, LDTA '04, 2004, pages 149-168.

RS-04-35 Jørgen Iversen. Type Checking Semantic Functions in ASDF. December 2004. 29 pp.

RS-04-34 Anders Møller and Michael I. Schwartzbach. The Design Space of Type Checkers for XML Transformation Languages. December 2004. 21 pp. Appears in Eiter and Libkin, editors, Database Theory: 10th International Conference, ICDT '05 Proceedings, LNCS 3363, 2005, pages 17-36.

RS-04-33 Aske Simon Christensen, Christian Kirkegaard, and Anders Møller. A Runtime System for XML Transformations in Java. December 2004. 15 pp. Appears in Bellahsene, Milo, Rys, Suciu and Unland, editors, Database and XML Technologies: Second International XML Database Symposium, XSym '04 Proceedings, LNCS 3186, 2004, pages 143-157. Supersedes the earlier BRICS report RS-03-29.

RS-04-32 Philipp Gerhardy. A Quantitative Version of Kirk's Fixed Point Theorem for Asymptotic Contractions. December 2004. 9 pp.

RS-04-31 Philipp Gerhardy and Ulrich Kohlenbach. Strongly Uniform Bounds from Semi-Constructive Proofs. December 2004. 31 pp.

RS-04-30 Olivier Danvy. From Reduction-Based to Reduction-Free Normalization. December 2004. $27 \mathrm{pp}$. Invited talk at the 4th International Workshop on Reduction Strategies in Rewriting and Programming, WRS 2004 (Aachen, Germany, June 2, 2004). To appear in ENTCS. 